\title{
Mercado Integrado Latinoamericano: diez años después de su creación. Un análisis bibliométrico para el periodo 2008-2021*
}

\author{
Integrated Latin American Market (MILA): Ten Years After its Creation. A \\ Bibliometric Analysis From 2008 to 2021
}

\author{
Juan Camilo Cardona-Montoya \\ Máster en Contabilidad, Auditoría y Mercado de Capitales, Universidad de San Buenaventura, \\ Medellín - Colombia, jucamo34@gmail.com
}

\begin{abstract}
Cómo citar / How to cite
Cardona-Montoya, J. C. (2022). Mercado Integrado Latinoamericano: diez años después de su creación. Un análisis bibliométrico para el periodo 2008-2021. Revista CEA, v. 8, n. 16, e1863.

https://doi.org/10.22430/24223182.1863
\end{abstract}

Recibido: 30 de abril de 2021

Aceptado: 13 de septiembre de 2021

\begin{abstract}
Resumen
El propósito de este artículo fue analizar las características de la producción investigativa y las temáticas abordadas entorno al Mercado Integrado Latinoamericano -MILA. Utilizando un análisis bibliométrico, a partir de 73 manuscritos publicados entre 2008 y 2021 en revistas indexadas en Scopus, Web Of Science y en otras bases de datos. Los resultados evidenciaron que, la/el docente Daniela Pérez Noreña/Luis Berggrun, la Universidad de Antioquia y Colombia se destacan como el autor, la organización y el país con los mayores niveles de publicación/de conexión. Asimismo, las temáticas de integración financiera e hipótesis de mercados eficientes sobresalen como las de mayor desarrollo científico alrededor de este acuerdo. Pese a lo anterior, y considerando, además, que la investigación sobre el Mercado Integrado Latinoamericano se está generando de manera fragmentada, con baja conexión entre autores, comunidades de trabajo y literatura previa sobre esta materia, este artículo contribuirá para que, a futuro, nuevas investigaciones puedan proseguir y profundizar de manera empírica en las líneas ya mencionadas y que hasta ahora son consideradas incipientes en el campo investigativo, pero de gran peso en la operatividad del mercado, o en aquellas que, por el contrario, reflejan un moderado avance, aunque con un pronunciado aislamiento entre sus publicaciones.
\end{abstract}

\footnotetext{
* Este artículo se deriva del proyecto titulado «Relación inversión-flujo de caja para las compañías cotizadas en los principales mercados de América» y ha financiado con recursos de la Universidad de San Buenaventura - Medellín.
} 
Palabras clave: Mercado Integrado Latinoamericano, Alianza del Pacífico, integración de los mercados de valores, análisis bibliométrico.

Clasificación JEL: F36, G10, G15.

\title{
Highlights
}

- La integración financiera y la hipótesis de mercados eficientes son algunas de las temáticas con mayor desarrollo y conexión científica alrededor del acuerdo Mercado Integrado Latinoamericano.

- La investigación sobre el Mercado Integrado Latinoamericano se ha generado de manera fragmentada, con baja conexión entre autores, universidades y literatura previa sobre esta materia.

- A partir de la segunda década de funcionamiento del Mercado Integrado Latinoamericano, la eficiencia transaccional será una oportunidad de producción investigativa.

\begin{abstract}
This paper analyzes the characteristics of the research production and the subjects addressed in the literature about the Integrated Latin American Market (MILA). A bibliometric analysis was carried out using 73 studies published between 2008 and 2021 in journals indexed in Scopus, Web of Science, and other databases. The results show that professors Daniela Pérez Noreña and Luis Berggrun, the Universidad de Antioquia, and Colombia are the authors, the organization, and the country with the highest levels of publication and co-authorship, respectively. Likewise, two topics, i.e., financial integration and the efficient market hypothesis, present the greatest scientific development in this field. However, research into the MILA has been conducted in a fragmented manner, with few connections between authors, research communities, and previous literature on this subject. This article can serve as a basis for future empirical research to further and more deeply explore these topics, which are still considered incipient research areas but are greatly important for the operational capacity of the market, or those in which moderate advances have been made but with a pronounced isolation between publications.
\end{abstract}

Keywords: Integrated Latin American Market, Pacific Alliance, stock market integration, bibliometric analysis.

JEL classification: F36, G10, G15.

\section{Highlights}

- In the literature about the Integrated Market Latin American, financial integration and the efficient market hypothesis are some of the topics that present the greatest development and most scientific connections.

- Research on the Integrated Latin American Market has been conducted in a fragmented manner, with few connections between authors, universities, and previous literature on this subject.

- After the second decade of the implementation of the Integrated Latin American Market, transactional efficiency will be a topic to research. 


\section{INTRODUCCIÓN}

En las dos últimas décadas, la estrategia de integración entre mercados bursátiles ha tomado una relevancia bastante significativa (Coleman et al., 2019), motivadas, en gran parte, por los tratados económicos entre países y regiones. En términos generales, en adquisiciones y alianzas se han configurado varias de estas integraciones. Muchos son los casos de éxito en casi todos los continentes, incluso algunos de ellos son considerados operaciones trasatlánticas. Con independencia de las figuras utilizadas, estos acuerdos entre bolsas incorporan varios beneficios a los actores de los mercados (Hennings Otoya, 2015; Lizarzaburu Bolaños et al., 2015), que van desde mayor competitividad y economías de escala para sus respectivas plazas, hasta representar un canal mucho más atractivo de financiación en el caso de los emisores y una expansión en la gama de productos para la estructuración e inversión en portafolios en cuanto a los bróker e inversores, respectivamente (Orozco Álvarez y Ramírez López, 2016).

La región latinoamericana no estuvo exenta de esta tendencia. A finales de mayo de 2011 entró en operación el Mercado Integrado Latinoamericano -MILA-, el cual fue constituido como un acuerdo de integración sin fusión entre las bolsas y depositarios de Colombia, Chile y Perú con la posterior incorporación de los participantes del mercado mexicano a mediados de 2014. Respaldado por la Alianza del Pacífico (AP), a través de los gobiernos y reguladores de los países integrantes, el MILA es considerado como una de las plazas bursátiles más grandes de Latinoamérica. Los beneficios descritos para las integraciones son también los perseguidos por el MILA, pero a lo largo de estos diez años de operación, ha debido enfrentarse a una serie de obstáculos y desafíos propios a las integraciones (Yepes-Ríos et al., 2015) y otros de carácter endógeno a su estructura. Estas implicaciones han despertado el interés de académicos y reguladores de la región por el desarrollo científico del MILA.

Dado lo anterior, este estudio tuvo por objetivos analizar las características de la producción investigativa, así como la evolución de las temáticas que se han desarrollado en torno al MILA. Para ello se utilizó un análisis bibliométrico a partir de 73 manuscritos seleccionados desde las bases de datos Scopus, Web of Science (WoS) y Google Académico para los años 2008-2021, tiempo en el cual se presentaron las negociaciones y acuerdos de integración (2008-2010) y el funcionamiento del mercado integrado (2011-2021). Con el apoyo de una hoja de cálculo y del software Gephi Versión 0.9.2, fueron calculados los descriptivos, gráficos y redes de coautoría y citas necesarios para este análisis.

Para responder a los objetivos planteados, este artículo presenta una revisión de la literatura respecto a las generalidades del MILA, comenzando con un recorrido por las principales integraciones a nivel mundial, para luego abordar a detalle varios estudios sobre el mercado integrado por grupos de temáticas, las cuales, por tener menor participación sobre el total de la muestra o presentar poca conexión entre sus publicaciones, no fueron analizadas a profundidad en el estudio bibliométrico. Seguidamente, se describe la metodología utilizada en la búsqueda, obtención y procesamiento de la muestra de manuscritos y se da paso a la presentación de los resultados del análisis bibliométrico. En el siguiente apartado se discuten los hallazgos encontrados y se sugieren futuras líneas de investigación. Finalmente, en la última sección se señalan las principales conclusiones y limitaciones de esta pesquisa. 
Mercado Integrado Latinoamericano: diez años después de su creación. Un análisis bibliométrico para el periodo 20082021

\section{MARCO TEÓRICO}

Las integraciones económicas entre países y regiones se han convertido en las últimas décadas en una dinámica estrategia de hacer negociaciones (Dicaprio y Santos-Paulino, 2011). Esta articulación de países, que va más allá de las fronteras y preferencias políticas, suele adoptar diversidad de formas, tales como zonas de preferencia, de libre comercio, uniones aduaneras, mercado común y comunidad económica (Vargas Pulido y Bayardo Martínez, 2013). Su configuración dependerá de los objetivos que se dirija a alcanzar, los cuales van direccionados a uno o varios de los siguientes ejes centrales: la libre movilidad de personas, capitales, bienes y servicios.

Bajo la iniciativa de la libre movilidad de capitales, los participantes de los mercados financieros, en especial las bolsas de valores, se han visto motivados por integrarse con sus pares con el fin de alcanzar mayor participación en los mercados bursátiles del exterior (Hennings Otoya, 2015; Palomino Selem, 2010). Dichas integraciones suelen adoptar dos tipos de negociaciones: adquisiciones y alianzas. Como ejemplos del primer tipo de integración, en el contexto internacional, se resalta la creación del mercado NYSE-Euronext, el cual es el resultado de la fusión de la bolsa de Nueva York con el conglomerado Euronext. Este último proviene de las sucesivas fusiones entre las bolsas de París, Ámsterdam, Bruselas, Lisboa, Oporto, Oslo, Dublín y el mercado de derivados de Londres (LIFFE, por sus siglas en inglés). En el entorno regional se destaca la fusión de las bolsas de valores de Bogotá, Medellín y Occidente en la actual Bolsa de Valores de Colombia.

Por otra parte, bajo la figura de alianzas se destaca principalmente los actuales esquemas de inversión colectiva, ejemplo de ello son los impulsados por Singapur, Malasia y Tailandia, tres países pertenecientes a la Asociación de Naciones del Sudeste Asiático (ASEAN, por sus siglas en inglés), y el promovido entre Hong Kong con Suiza, Francia, Reino Unido, Luxemburgo y con la misma China continental. Dentro de este tipo de esquemas de inversión se destaca, además, el impulsado por Australia, Japón, Nueva Zelanda, Tailandia y la República de Corea, todos ellos asociados al Foro de Cooperación Económica Asia-Pacífico (APEC, por sus siglas en inglés) (Rodríguez Aranda, 2014; Basnet et al., 2015). Respecto a la figura de alianzas entre mercados de valores, pero sin representar acuerdos de fusión entre ellos, se destacan el denominado Stock Connect, en el que se han incorporado sucesivamente los mercados bursátiles de Hong Kong y China continental, y el Mercado Integrado Latinoamericano o MILA, conformado desde mediados de 2011 por las bolsas y los depósitos centrales de Chile, Colombia y Perú, con la posterior incorporación de los participantes bursátiles de México a finales de 2014 (Mellado y Escobari, 2015).

Simultáneamente, con la propuesta del mercado MILA, motivada por el sector privado bursátil, una nueva iniciativa de integración macro fue impulsada por los gobiernos de los cuatro países en mención. Bajo el nombre de Alianza del Pacífico, dicho bloque regional busca desarrollar los cuatro ejes centrales aclarados al inicio de esta sección, estos son la libre circulación de bienes, servicios, capitales y personas. En particular, para dar marcha a su pilar de libre movilidad de capitales, la AP respaldó la iniciativa privada por la creación del Mercado Integrado (Duarte Herrera et al., 2014), al tiempo que las instituciones reguladoras y supervisoras del mercado de valores de los países participantes también refrendaron la creación del mercado integrado (Santillán-Salgado et al., 2017). Tras la suscripción de un memorando de entendimiento en 2009, y otro en 2010, estas instituciones se comprometieron a intercambiar información y a cooperar y coordinar esfuerzos entre ellas, así como también a seleccionar un modelo de enrutamiento intermediado de órdenes que incorporara 
la totalidad de los procesos transaccional y postransaccional en manos de la bolsa en donde originalmente se listara el título.

Dicho modelo de enrutamiento intermediado, adoptado para operar en el mercado integrado, permite a los inversores ejecutar sus órdenes sobre valores localizados en los países miembros, únicamente acudiendo a sus corredores locales, quienes, bajo la figura de enrutadores autorizados para tranzar en el MILA, se conectan con sus contrapartes en la nación de destino, para que estos últimos registren la oferta de compra en su correspondiente plaza bursátil (Hurtado Briceño, 2017). De la misma manera, los restantes procesos de compensación, liquidación y custodia de valores continuarán unificados en el mercado de cotización del título, cumpliendo con la regulación del país respectivo (Baquero-Herrera, 2013).

De acuerdo con Hennings Otoya (2015), Lizarzaburu Bolaños et al. (2015) y Espinoza Méndez et al. (2017), esta novedosa estrategia de integración en la región (el MILA) representa amplias oportunidades de crecimiento para los diferentes actores, las cuales van desde una mayor diversificación y liquidez en la conformación de portafolios para el inversor hasta representar un mercado alterno de financiación con mejores condiciones para los emisores de valores (Hurtado Briceño, 2017; Orozco Álvarez y Ramírez López, 2016). Pero no todo son beneficios, aparte de los choques regionales y efectos indirectos y de contagio, que la literatura previa ha identificado como posibles riesgos derivados de la integración entre bolsas (Yepes-Ríos et al., 2015), el mercado MILA aún adolece de ciertos aspectos relacionados con la heterogeneidad en los costos de transacción explícitos entre países, que, de algún modo, podrían sesgar los flujos para la inversión aventajando a un grupo de empresas, mercado o sector en particular (Palomino Selem, 2010). Aspectos como la falta de una divisa homogénea para transar, que obliga a recurrir al tipo de cambio de cada moneda local respecto al dólar, la ausencia de una política tributaria homologada entre los países miembros (Palomino Selem, 2010), así como la necesidad de unificar las políticas de intermediación y corretaje, que normalice los cobros y las comisiones por estas operaciones, probablemente impacten el desempeño del mercado integrado.

Por lo anterior, MILA se ha convertido en objeto de investigación por parte de los organismos reguladores e investigadores de la región y por fuera de ella. A lo largo de estos años de funcionamiento, varios estudios han contrastado algunos de sus beneficios, riesgos y desafíos previamente indicados, $y$, a su vez, estos han permitido identificar un conjunto de líneas de investigación, algunas con mayor profundidad y desarrollo y otras que posiblemente serán exploradas durante los años que vienen. En este sentido, la integración financiera seguida de lejos por la hipótesis de mercados eficientes (HME) son los tópicos que mayor profundidad y conexión han alcanzado alrededor de este acuerdo. Esta última línea en particular, a pesar de abordarse con mayor detalle al final de los resultados de este estudio, conviene mencionar dentro de ella a HernándezGamarra et al., (2015), Meneses Cerón y Pérez Pacheco (2020) y Ramírez (2016). Si bien estos estudios se caracterizan por encontrarse al margen de la literatura de HME, que se está tejiendo alrededor de los mercados del MILA, sí que le hacen una significativa contribución a la misma en términos de fortalecer la evidencia previa, con el empleo de técnicas, metodologías y alcances alternativos a los tradicionalmente observados.

Otras temáticas, por el contrario, permanecen casi inexploradas en la literatura del MILA y probablemente serán desarrolladas en la siguiente década, entre ellas se destacan la liquidez, el tipo 
de cambio y las reglas fiscales al interior de la integración. Estos factores, catalogados por Hurtado Briceño (2017) como de relevancia para la eficiencia transaccional del mercado, probablemente han motivado la aparición de ciertas publicaciones un tanto dispersas entre sí y del resto de literatura. En representación de la primera línea, se reportan los estudios de Fuenzalida et al. (2017) y Agudelo et al. (2012). Estos, más que analizar empíricamente el impacto real que ha tenido el acuerdo en los costos de transacción implícitos -como una de las medidas de liquidez en cada mercado/título participante-, examinan, en el primero de los manuscritos, si dentro del mercado integrado existe alguna prima asociada a la iliquidez de las acciones, mientras que en el segundo, realizan una proyección econométrica de cuánto podría ser el ahorro respecto a este tipo de costos si se incrementara el volumen negociado en cada mercado participante, a propósito de la entrada en vigor del MILA.

Dentro de la segunda línea por explorar, es decir, la relacionada con el tipo de cambio y su posible impacto en las negociaciones dentro del mercado integrado, Farfán et al. (2017) y Sosa et al. (2018) siguen de cerca este propósito, tras evaluar en sus respectivos estudios cuál es la relación existente entre los mercados de acciones y de divisas. Respecto a la tercera temática, la relacionada con la heterogeneidad y complejidad de las reglas fiscales al interior de los países miembros, ha habido un importante avance, al menos desde un alcance exploratorio-descriptivo y de simulación, puesto que, Gil-Solano (2020), Hennings Otoya (2015) y Maruy et al. (2015) se aproximan a una comprensión de todo el entramado fiscal por cada país integrante en lo que respecta a convenios de no doble tributación y a impuestos a las ganancias de capital por concepto de dividendos y de compra-venta de acciones a través de la plataforma MILA.

A diferencia de la temática anterior, otros tópicos han sido abordados con mayor profundidad alrededor del MILA, aunque se advierte que se caracterizan por existir poca conexión entre sí con respecto a los referentes teóricos. Como primer caso, se hace mención a un conjunto de estudios que, en términos agregados, se identifican por proponer, contrastar y estructurar portafolios eficientes de inversión a través de un amplio abanico de posibles modelos y de títulos disponibles en el mercado integrado, entre estos se destacan Ariza et al. (2012), García et al. (2020), García et al. (2019), Luna-Ramírez y Agudelo (2019), Morales León y Vélez Molano (2020), Núñez-Mora et al. (2017), Pérez Sosa et al. (2016) y Uribe Gil y Mosquera López (2014). En segundo lugar, se ubican los estudios que tienen como finalidad identificar aquellos factores determinantes de los retornos en el mercado del MILA. Las publicaciones hechas por Berggrun et al. (2016), Carmona Muñoz y Vera Leyton (2017), Chávez Muñoz et al. (2020), Gutiérrez Betancur et al. (2017), Gutiérrez Castañeda et al. (2020b), Idrogo (2019), Peña Figueroa (2019), Pérez Noreña et al. (2020) y Sandoval Álamos et al. (2015), pueden ser atribuidas a este grupo.

Finalmente, tomando en consideración las manifestaciones hechas por Leraul (2016), respecto a asumir como factores de preocupación y desmotivación de los participantes del MILA a las problemáticas relacionadas con la diversidad en las reglas de buen gobierno, los escasos requerimientos de información adicional que deben suministrar los emisores de valores y la dificultad de acceso a este material por parte de los inversores, es que se configura la siguiente temática de investigación. Bajo el concepto de reportes integrados, este nuevo grupo se destaca por analizar dicho tópico desde múltiples aristas, además de caracterizarse por contar con bastante participación de publicaciones, aunque adolece de conexión entre ellas, tal y como sucede con varias de las temáticas que se están adelantando en torno al acuerdo. 
Entre los subtemas analizados por esta línea (la de reportes integrados), se destacan el dictamen de auditoría/el reporte de sostenibilidad. Estos informes son examinados desde sus aspectos de estructura y presentación (Zamarra-Londoño et al., 2020; Giraldo López et al., 2018) y el impacto de sus contenidos en el desempeño empresarial (Zamarra Londoño et al., 2021; Jaramillo Arango et al., 2020); adicionalmente, Zamarra Londoño et al. (2021) indagan por los factores que determinan el conseguir un dictamen del auditor sin salvedades, mientras que para el segundo tipo de reporte, Garzón Jiménez y Zorio-Grima (2021) analizaran por el impacto de este en los problemas de información asimétrica, al tiempo que Suárez-Rico et al. (2019) averiguaran por los determinantes en la preferencia por el uso de redes sociales como medio de divulgación de las acciones de responsabilidad social corporativa contenidas en dicho informe.

Dentro de la línea de reportes integrados también han sido protagonistas los subtemas de gobierno corporativo y calidad de la información financiera. En el primero, los estudios se han enfocado principalmente en comparar los mecanismos que forman parte de este sistema entre los países miembros (Gallardo Loaiza, 2014) y en develar el impacto de varias de las prácticas y de las características de buen gobierno sobre el desempeño empresarial (Acevedo Arango et al., 2020; Améstica Rivas et al., 2021; Torres-Cano y Correa-Mejía, 2021), entre ellas, la fuerza laboral femenina, considerada en toda su extensión dentro de la organización (Álvarez-Flores et al., 2020). El último de los subtemas, por su parte, ha tenido un alcance más limitado. Sus estudios han indagado por el impacto de esta cualidad del reporte financiero, así como también del efecto de la entrada en vigor del MILA y de la aplicación de las IFRS sobre los problemas de asimetría en la información (Muñoz Mendoza et al., 2021). De la misma manera, los factores determinantes de la calidad del reporte, entre los que sobresalen algunas prácticas de bueno gobierno (Perafán Peña, 2018), también han sido objeto de análisis dentro del acuerdo.

\section{METODOLOGÍA}

Para desarrollar los objetivos de este estudio, de analizar las características de la producción científica y la evolución de las temáticas desarrolladas entorno al MILA, fue empleado un análisis bibliométrico basado principalmente en técnicas descriptivas, gráficos y redes de coautoría y citas. Según Durisin et al. (2010), y más recientemente Arias-Ciro (2020), los análisis de tipo bibliométrico permiten determinar la situación actual y el progreso de una disciplina en particular, además de identificar tendencias en el conocimiento (Arduini y Zanfei, 2014; Carvalho et al., 2013; Sancho, 1990).

Este tipo de análisis, que utiliza técnicas matemáticas y estadísticas para estudiar los patrones de publicación y comunicación en la distribución de la información (Durieux y Gevenois, 2010), ya ha sido validado previamente por la literatura y utilizado por múltiples disciplinas en diversos campos del conocimiento, por ejemplo, los estudios desarrollados por Pineda Ospina (2015) y Venable et al. (2014), para los sectores de alimentos y salud, respectivamente, o los presentados recientemente para las áreas de responsabilidad social empresarial (Jaén et al., 2018) y Gobierno corporativo universitario (King et al., 2019). Para el caso particular del MILA, este sería el primer estudio del que se tenga conocimiento en reportar el uso de un análisis bibliométrico sobre la producción científica en esta materia, a pesar de reconocer que, para este mismo acuerdo, Ortegón Rojas y Torres Castro (2016) realizaron una revisión de la literatura, y aunque de una forma somera y poco profunda, analizaron nueve manuscritos identificados sobre este tema entre el año 2011 y principios de 2016. 
Ahora bien, para proceder con la aplicación del análisis bibliométrico en este estudio, se recopiló con anterioridad la producción investigativa desarrollada alrededor del acuerdo. Para esto fue necesario el uso de palabras clave durante el proceso de búsqueda en las bases de datos (BD) de mayor prestigio internacional: Scopus y Web Of Science (WoS). La consulta fue realizada demarcando la exploración bajo el filtro de búsqueda por temas, títulos, resúmenes y palabras clave. Adicionalmente, a priori, considerando que los investigadores latinoamericanos se caracterizan por publicar frecuentemente sus avances en las propias revistas locales, muchas de ellas no indexadas en las anteriores BD, se hizo necesario ampliar la exploración a otras bases de datos a partir de la indexación de las revistas en Google Académico. Sin embargo, en cuanto al proceso de selección y depuración de los documentos provenientes de estas últimas, se tuvo un cierto nivel de cautela, teniendo cuidado de no incluir aquellos documentos caracterizados por no seguir un proceso riguroso de investigación científica, como lo son los informes técnicos y de avances sobre la integración.

Retomando ahora los detalles del proceso de búsqueda en las BD, las palabras clave utilizadas fueron: «Mercado Integrado Latinoamericano», "MILA», "Latin American Integrated Market», "Mercado Integrado», «Integrated Market» y "LAIM». La consulta fue realizada a finales de septiembre de 2020 y actualizada a finales de mayo de 2021, además, se fijó el rango de análisis 2008-2021, dado que, previo a la implementación y puesta en marcha del MILA, que fue a mediados de 2011, probablemente la literatura ya había comenzado a enfocar su mirada hacia este mercado durante los años de acuerdos, negociaciones y estructuración por parte de sus artífices, es decir, entre 20082010.

De la anterior búsqueda por palabras clave y la consiguiente revisión de los títulos y del resumen de cada manuscrito, se logró depurar aquellas publicaciones que realmente no exploraron o profundizaron en algún aspecto del MILA. Es así como se seleccionó un total de 73 manuscritos para el análisis, divulgados en 59 fuentes de publicación distintas. De estos 73, cuatro provienen desde cuatro revistas indexadas en Scopus; once, desde nueve revistas recogidas en WoS ; diecinueve más, desde trece fuentes incluidas en ambas bases de datos (Scopus y WoS); y las restantes 39 publicaciones fueron identificadas en 33 medios incorporados en otras bases de datos.

Posterior a la anterior selección, fue elaborada una matriz de artículos en una hoja de cálculo. Las características de cada uno fueron capturadas y clasificadas según algunos de los tipos de indicadores definidos por Ardanuy (2012), estos son, de producción, de forma y contenido, y de impactocolaboración. En el primero de estos grupos, las mediciones son hechas y desagregadas en lo concerniente a la fuente de publicación, de institución de origen y de autor. Para ello, se recopila información acerca del año de publicación, nombre, país de domicilio y/e bases de datos/institución en donde se encuentra indexada/afiliado cada fuente de publicación/autor. El segundo conjunto de medidores reúne las características de idioma de publicación, temática abordada, metodología empleada y tipo de documento. Finalmente, el tercer conglomerado de medidas, que lo componen las conexiones hechas entre autores y citaciones entre artículos, aparte de ser visto como una expresión de visibilidad e impacto generado entre los investigadores y las publicaciones, también puede ser asumido como una señal del trabajo conjunto a este nivel de detalle. A partir de la matriz de datos recopilados, y con el apoyo de una hoja de cálculo y del software Gephi Versión 0.9.2, se procedió a calcular y a generar los descriptivos, gráficos y redes de coautoría y citas, los cuales son presentados y analizados en la siguiente sección. 


\section{RESULTADOS}

Si bien, el MILA entró oficialmente en operación a finales del mes de mayo de 2011, durante su periodo de negociaciones y acuerdos (2008-2010) ya se dejaba entre ver una literatura naciente que describía las características de este mercado. La primera publicación de carácter investigativo de la que se tiene evidencia data del año 2010 (Figura 1), bajo patrocinio peruano fue escrita por Palomino Selem y publicada por Pensamiento Crítico, una revista domiciliada en el mismo país de origen. El interés por investigar las particularidades del mercado MILA vuelve a cobrar importancia en publicaciones del año 2012, esta vez por autores y patrocinio principalmente de Colombia. Desde ese año, las divulgaciones se han mantenido de forma ininterrumpida e incremental respecto de lo evidenciado previamente; sin embargo, para el periodo que abarca este análisis (2008-2021), únicamente se identificaron 73 manuscritos sobre esta materia en las tres bases de datos definidas en la metodología (Scopus, WoS y Google Académico).

Continuando con el análisis de la producción investigativa por periodo, el año 2020 registra los mayores niveles de publicación hasta la fecha, concentrando un poco más de la quinta parte del total de los manuscritos sobre el MILA. Precisamente, las publicaciones de autores e instituciones colombianas, en cabeza de la Universidad de Antioquia, son las que conducen a este resultado. Seguidamente, el 2016 se convierte en el periodo con el segundo nivel de producción científica más alto, año en el que el repunte de las publicaciones, más allá de los aportes que los autores e instituciones colombianas le hacen habitualmente a esta literatura, pertenecen a los primeros productos publicados de origen y patrocinio mexicano. Este hecho coincide con el desarrollo de proyectos investigativos entre los años 2014-2016 a causa de la adhesión de México, a finales del 2014, al mercado integrado. A partir de 2017 se evidenció un paulatino debilitamiento en el número de artículos identificados, sin embargo, las divulgaciones del sexenio 2015-2020 concentraron casi el $80 \%$ de lo publicado y se ubicaron por encima de la media (6,6 artículos por año), con excepción del año 2018, en el cual se registró un total de seis publicaciones sobre el mercado MILA.

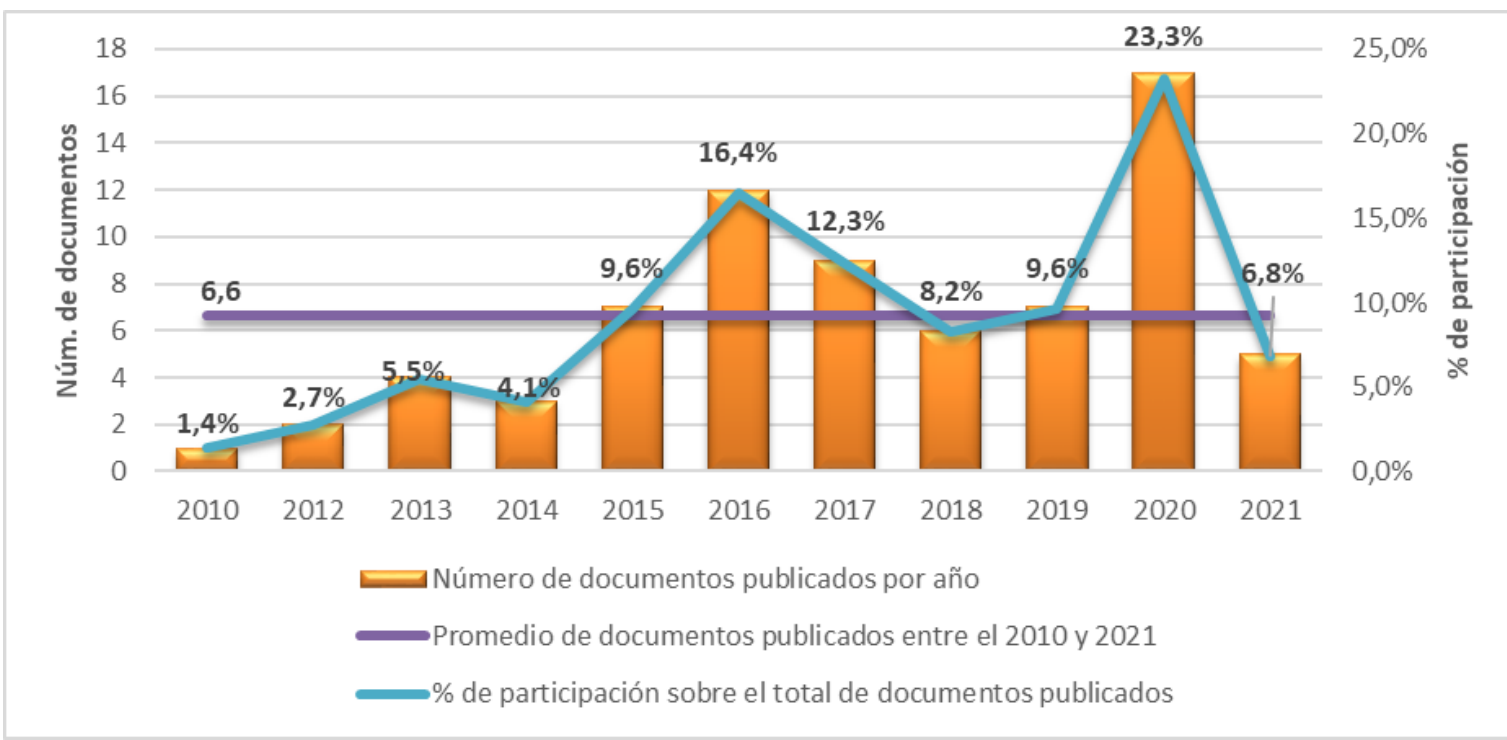

Figura 1. Distribución temporal de los documentos publicados sobre el mercado MILA, periodo 2010-2021

Figure 1. Distribution of documents on the MILA published between 2010 and 2021

Fuente: elaboración propia con base en la información reportada por Scopus, WoS y Google Académico. 
Además de un análisis por año, se consideró agrupar los manuscritos identificados según su idioma de publicación (Tabla 1). En este sentido, dado que la plataforma del MILA reúne los mercados bursátiles de cuatro de las principales economías más prometedoras de la región, en las que su lengua oficial es el español, es de esperarse una participación mayoritaria de las publicaciones en dicha lengua, aunque los documentos divulgados en el idioma inglés representan un peso importante. Con cerca del $40 \%$ de contribución sobre el total, los artículos publicados en dicho idioma no sólo reflejan que el mercado integrado no es un tema exclusivo de la región latina y que se discute como tema central en los diálogos internacionales, sino que también refleja el esfuerzo, principalmente, de las organizaciones, universidades e investigadores de la región por publicar en revistas con indexación internacional, las cuales, en su gran mayoría, publican los manuscritos en el lenguaje anglosajón.

Tabla 1. Distribución de los documentos sobre el mercado MILA según su idioma de publicación Table 1. Distribution of documents about the MILA by language

\begin{tabular}{ccc}
\hline $\begin{array}{c}\text { Idioma de la } \\
\text { publicación }\end{array}$ & $\begin{array}{c}\text { Número de documentos } \\
\text { publicados }\end{array}$ & \% de participación \\
\hline Español & 44 & $60,3 \%$ \\
\hline Inglés & 29 & $39,7 \%$ \\
\hline Total & 73 & $100 \%$ \\
\hline
\end{tabular}

Ahora bien, como es tendencia en los estudios científicos, gran parte de las producciones investigativas sobre el mercado integrado también son publicadas en revistas. De acuerdo con la Figura 2, el $82 \%$ de los manuscritos se lista como artículos de investigación mientras que el $6 \%$ son documentos de reflexión. Las publicaciones hechas como capítulos de libro, artículos de conferencia y documentos de trabajo (working papers), si bien son poco relevantes, comienzan a cobrar importancia en términos agregados dentro del total con un $10 \%$ de participación conjunta.

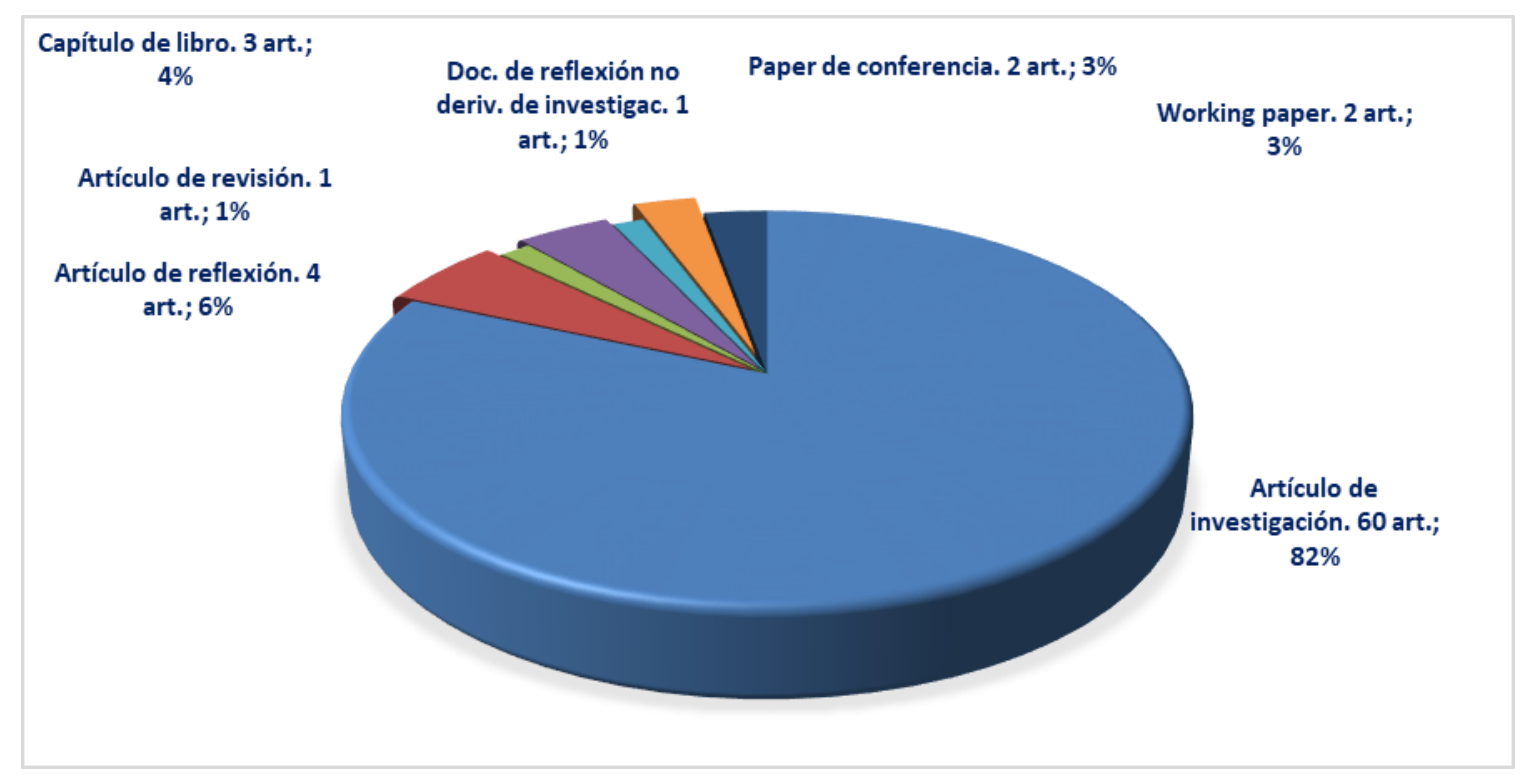

Figura 2. Distribución de las publicaciones sobre el MILA según el tipo de documento

Figure 2. Distribution of publications on the MILA classified by document type Fuente: elaboración propia con base en la información reportada por Scopus, WoS y Google Académico. 
Con relación al nivel de acreditación de las fuentes o medios, en los cuales se prefiere publicar los resultados de las investigaciones sobre el MILA, la Tabla 2 revela que más de la mitad de los documentos, específicamente el 53,4\% de ellos, son publicados en revistas indexadas en otras bases de datos distintas a WoS y Scopus. El segundo lugar de preferencia para publicar corresponde a las revistas tipo WoS y Scopus-WoS, las cuales alcanzan un 41,1\% de participación agregada, destacándose dentro de este último grupo (Scopus-WoS) las contribuciones hechas en revistas ubicadas en los cuartiles 2 y 4 de Scopus.

Tabla 2. Distribución de documentos sobre el mercado MILA según el nivel de acreditación de la fuente de publicación

Table 2. Distribution of documents about the MILA by journal quartile

\begin{tabular}{|c|c|c|}
\hline Base de datos de indexación & Número de documentos & \% de participación \\
\hline \multicolumn{3}{|l|}{ Revistas indexadas en Scopus } \\
\hline Scopus-Q1 & 0 & $0,0 \%$ \\
\hline Scopus-Q2 & 1 & $1,4 \%$ \\
\hline Scopus-Q3 & 1 & $1,4 \%$ \\
\hline Scopus-Q4 & 2 & $2,7 \%$ \\
\hline Total, revistas indexadas en Scopus & 4 & $5,5 \%$ \\
\hline \multicolumn{3}{|l|}{ Revistas indexadas en Scopusy WoS } \\
\hline Scopus-Q1 & 0 & $0,0 \%$ \\
\hline Scopus-Q2 & 9 & $12,3 \%$ \\
\hline Scopus-Q3 & 3 & $4,1 \%$ \\
\hline Scopus-Q4 & 7 & $9,6 \%$ \\
\hline $\begin{array}{l}\text { Total, revistas indexadas en Scopusy } \\
\text { WoS }\end{array}$ & 19 & $26,0 \%$ \\
\hline WoS & 11 & $15,1 \%$ \\
\hline Otras bases de datos & 39 & $53,4 \%$ \\
\hline Total general & 73 & $100 \%$ \\
\hline
\end{tabular}

Al categorizar cada fuente de publicación según su BD de indexación, se prosiguió con la siguiente jerarquía: primero fueron categorizadas como revistas tipo Scopusa aquellas indexadas en Scimago Journal \& Country Rank. De no estarlo, se prosiguió con su búsqueda en WoS y, de no encontrarse en las dos anteriores, fue clasificada en la categoría de Otras bases de datos a partir de su búsqueda en Google Académico, lo cual permitió identificar su ubicación en BD como Dialnet, Ebsco, Latindex, Proquest, Redalyc, Scielo, Publindex, entre otras. Para dirimir los casos en los cuales una revista fuera indexada tanto en Scopus como en WoS, se respetaron sus dos indexaciones. El cuartil añadido a las fuentes recogidas en Scopus fue considerado según el año en que ha sido publicado cada manuscrito y no hace referencia al cuartil de ubicación actual de la misma. Como aún no se tiene disponible en Scimago Journal \& Country Rank los resultados de desempeño para el periodo 2021, fue clasificado el cuartil de algunas de estas, que reportaron publicación en dicho año con base en su desempeño obtenido en 2020. 
Mercado Integrado Latinoamericano: diez años después de su creación. Un análisis bibliométrico para el periodo 20082021

Profundizando ahora en las características de las fuentes o medios de publicación, la Tabla 3 hace referencia al domicilio de las instituciones editoras de dichas fuentes. Según esta, más del 68,5\% de los documentos publicados sobre el mercado integrado son presentados en revistas con domicilio en Latinoamérica. Dentro de esta región, la mayor participación se registra en los medios de origen colombiano (43,8\%) y mexicano (11,0\%). Seguidamente, con un 20,5\% de aporte, Europa, en cabeza de Reino Unido (11,0\%) y España $(4,1 \%)$ principalmente, se ubica como el siguiente domicilio de revistas en las que se presentan artículos sobre el MILA. Las investigaciones acerca del acuerdo de integración también se han divulgado en otras regiones del mundo; es así como las fuentes americanas, por ejemplo, han captado el $8,2 \%$ de estas publicaciones a la fecha, mientras que en las asiáticas se ha evidenciado un leve repunte de publicaciones que abordan a este mercado.

Tabla 3. Distribución de documentos sobre el mercado MILA por país/región de domicilio registrado de la fuente de publicación

Table 3. Distribution of documents about the MILA by country/region of the publication

\begin{tabular}{|c|c|c|}
\hline País de domicilio de la fuente de publicación & Número de documentos & \% de participación \\
\hline Chile & 2 & $2,7 \%$ \\
\hline Colombia & 32 & $43,8 \%$ \\
\hline Cuba & 1 & $1,4 \%$ \\
\hline México & 8 & $11,0 \%$ \\
\hline Perú & 6 & $8,2 \%$ \\
\hline Venezuela & 1 & $1,4 \%$ \\
\hline Total Latinoamérica & 50 & $68,5 \%$ \\
\hline Estados Unidos & 6 & $8,2 \%$ \\
\hline Total Angloamérica & 6 & $8,2 \%$ \\
\hline España & 3 & $4,1 \%$ \\
\hline Holanda & 1 & $1,4 \%$ \\
\hline Lituania & 2 & $2,7 \%$ \\
\hline Reino Unido & 8 & $11,0 \%$ \\
\hline Ucrania & 1 & $1,4 \%$ \\
\hline Total Europa & 15 & $20,5 \%$ \\
\hline India & 1 & $1,4 \%$ \\
\hline Turquía & 1 & $1,4 \%$ \\
\hline Total Asia & 2 & $2,7 \%$ \\
\hline Total general & 73 & $100 \%$ \\
\hline
\end{tabular}

Ahondando en cuáles son aquellas fuentes en las que se han publicado más de un documento relacionado con el mercado integrado, la Tabla 4 revela que, con excepción de las revistas Apuntes Contables, Finanzas y Política Económica y Emerging Markets Finance and Trade, que han publicado tres artículos por cada una, en sólo ocho fuentes de publicación se han presentado individualmente 
un máximo de dos publicaciones relacionadas con la integración. En términos de concentración de las divulgaciones, en estas once fuentes se agrupan cerca del $34 \%$ de los manuscritos (25 documentos), mientras que el restante $66 \%$ (48 documentos) se encuentra disperso en 48 medios, de los cuales, veinte son de procedencia colombiana (20 documentos), y trece de otros países (13 documentos), registran la mayor porción dentro de las publicaciones dispersas.

En términos de participación de instituciones y autores, que pudiese explicar el elevado número de artículos sobre el MILA concentrado en estas once fuentes, cabe resaltar que en dos de ellas se presenta hasta dos publicaciones por cada una, bajo el patrocinio de la Universidad de Antioquia, este es el caso de las revistas Suma de Negocios y Apuntes Contables. En esta última, en particular, se evidencia que dos de los tres manuscritos publicados que se tienen registro acerca del acuerdo fueron bajo la autoría de las investigadoras Belky Esperanza Gutiérrez y Daniela Pérez Noreña, adscritas a dicha institución colombiana.

Tabla 4. Principales fuentes de publicación sobre el mercado MILA Table 4. Main sources of publications on the MILA

\begin{tabular}{cc}
\hline Taente de publicación & Número de documentos \\
\hline Apuntes Contables & 3 \\
\hline Revista Finanzas y Política Económica & 3 \\
\hline Emerging Markets Finance and Trade & 3 \\
\hline Contaduría Universidad de Antioquia & 2 \\
\hline Sinapsis & 2 \\
\hline Suma de Negocios & 2 \\
\hline Análisis Económico & 2 \\
\hline Estocástica: finanzas y riesgo & 2 \\
\hline Journal of Economial Universidad de Guadalajara & 2 \\
\hline $\begin{array}{c}\text { Science } \\
\text { Academia Revista Latinoamericana de } \\
\text { Administración }\end{array}$ & 2 \\
\hline Fuente: elaboración propia.
\end{tabular}

Por otra parte, al indagar por las características de los autores que publican acerca del MILA, la Tabla 5 detalla la región y país de domicilio de las afiliaciones institucionales de estos. Según esta Tabla, la producción investigativa se concentra en un total de doce países. Con un dominante $89 \%$ de participación, las organizaciones latinoamericanas lideran esta línea, y, a pesar de que los cuatro países que conforman la integración ostentan la mayor parte del patrocinio, se destaca el amplio porcentaje aportado por las organizaciones colombianas, las cuales acumulan casi la mitad de toda la producción. El resto de los patrocinios fuera de los países miembros del mercado integrado no son significativos, sin embargo, fuera de Latinoamérica, algunas instituciones domiciliadas en España y en los Estados Unidos se hacen a un 4,3\% y 3,2\% de participación institucional, respectivamente. 
Mercado Integrado Latinoamericano: diez años después de su creación. Un análisis bibliométrico para el periodo 20082021

Tabla 5. Participación en publicaciones sobre el mercado MILA, distribuida por país/región de domicilio de las afiliaciones institucionales de los autores

Table 5. Number of publications on the MILA classified by country/region of the authors' institutional affiliations

\begin{tabular}{|c|c|c|}
\hline $\begin{array}{l}\text { País de domicilio de las afiliaciones } \\
\text { institucionales }\end{array}$ & $\begin{array}{c}\text { Participaciones en } \\
\text { documentos }\end{array}$ & \% de participación \\
\hline Argentina & 1 & $1,1 \%$ \\
\hline Chile & 14 & $14,9 \%$ \\
\hline Colombia & 44 & $46,8 \%$ \\
\hline Ecuador & 1 & $1,1 \%$ \\
\hline México & 14 & $14,9 \%$ \\
\hline Perú & 9 & $9,6 \%$ \\
\hline Venezuela & 1 & $1,1 \%$ \\
\hline Total Latinoamérica & 84 & $89,4 \%$ \\
\hline Estados Unidos & 3 & $3,2 \%$ \\
\hline Total Angloamérica & 3 & $3,2 \%$ \\
\hline España & 4 & $4,3 \%$ \\
\hline Lituania & 1 & $1,1 \%$ \\
\hline Portugal & 1 & $1,1 \%$ \\
\hline Total Europa & 6 & $6,4 \%$ \\
\hline China & 1 & $1,1 \%$ \\
\hline Total Asia & 1 & $1,1 \%$ \\
\hline Total general & 94 & $100 \%$ \\
\hline
\end{tabular}

Respecto a cuáles son específicamente aquellas instituciones por cada país que han financiado el mayor número de publicaciones sobre el MILA, la Tabla 6 desvela que, de las ochenta organizaciones identificadas, sólo 27 (33,7\%) han patrocinado la publicación de más de un artículo. En principio, atendiendo al número de centros universitarios por jurisdicción (lado izquierdo de la Tabla), se evidencia una mayor cuota de universidades colombianas que participan en mínimo dos documentos acerca del mercado integrado, exactamente son doce de ellas, seguido por México, Perú y Chile, con cuatro cada uno, España con dos y Estados Unidos con tan sólo una institución. Atendiendo ahora al número de manuscritos por cada organización (lado derecho de la Tabla), la Universidad de Antioquia, el EGADE Business School, del Tecnológico de Monterrey y la Universidad Icesi, llevan la delantera patrocinando siete, seis y cinco publicaciones, respectivamente. Otros centros de educación relevantes por financiar un número igual a cuatro artículos son las universidades de Concepción (Chile) y EAFIT (Colombia). 
Tabla 6. Principales afiliaciones institucionales de los autores que investigan sobre el MILA, clasificadas por país de domicilio

Table 6. Most common institutional affiliations of authors researching the MILA classified by country

\begin{tabular}{|c|c|c|c|}
\hline $\begin{array}{l}\text { Núm. de } \\
\text { instituciones }\end{array}$ & Afiliación institucional & $\begin{array}{l}\text { Participaciones } \\
\text { en documentos }\end{array}$ & $\begin{array}{l}\text { \% de } \\
\text { participación }\end{array}$ \\
\hline 1 & Universidad de Concepción & 4 & $3,2 \%$ \\
\hline 1 & Independiente & 2 & $1,6 \%$ \\
\hline 1 & Universidad Católica de la Santísima Concepción & 2 & $1,6 \%$ \\
\hline 1 & Universidad Santo Tomás & 2 & $1,6 \%$ \\
\hline 10 & $\begin{array}{l}\text { Otras instituciones chilenas con una participación como máximo en } \\
\text { publicaciones sobre el MILA }\end{array}$ & 10 & $7,9 \%$ \\
\hline 14 & Total de participaciones universidades chilenas & 20 & $15,9 \%$ \\
\hline 1 & Universidad de Antioquia & 7 & $5,6 \%$ \\
\hline 1 & Universidad Icesi & 5 & $4,0 \%$ \\
\hline 1 & Universidad EAFIT & 4 & $3,2 \%$ \\
\hline 1 & Universidad Militar Nueva Granada & 3 & $2,4 \%$ \\
\hline 1 & Independiente & 2 & $1,6 \%$ \\
\hline 1 & Pontificia Universidad Javeriana & 2 & $1,6 \%$ \\
\hline 1 & Universidad de la Salle & 2 & $1,6 \%$ \\
\hline 1 & Universidad de los Andes & 2 & $1,6 \%$ \\
\hline 1 & Universidad de Medellín & 2 & $1,6 \%$ \\
\hline 1 & Universidad Nacional de Colombia & 2 & $1,6 \%$ \\
\hline 1 & Universidad Piloto de Colombia & 2 & $1,6 \%$ \\
\hline 1 & Universidad Pontificia Bolivariana & 2 & $1,6 \%$ \\
\hline 25 & $\begin{array}{l}\text { Otras instituciones colombianas con una participación como máximo } \\
\text { en publicaciones sobre el MILA }\end{array}$ & 25 & $19,8 \%$ \\
\hline 37 & Total de participaciones universidades colombianas & 60 & $47,6 \%$ \\
\hline 1 & University of Texas & 2 & $1,6 \%$ \\
\hline 1 & $\begin{array}{l}\text { Otras instituciones americanas con una participación como máximo en } \\
\text { publicaciones sobre el MILA }\end{array}$ & 1 & $0,8 \%$ \\
\hline 2 & Total de participaciones universidades americanas & 3 & $2,4 \%$ \\
\hline 1 & EGADE Business School (Tecnológico de Monterrey) & 6 & $4,8 \%$ \\
\hline 1 & CONACYT-CIDE & 2 & $1,6 \%$ \\
\hline 1 & Universidad Autónoma Metropolitana & 2 & $1,6 \%$ \\
\hline 1 & Universidad Nacional Autónoma de México & 2 & $1,6 \%$ \\
\hline 6 & $\begin{array}{l}\text { Otras instituciones mexicanas con una participación como máximo en } \\
\text { publicaciones sobre el MILA }\end{array}$ & 6 & $4,8 \%$ \\
\hline 10 & Total de participaciones universidades mexicanas & 18 & $14,3 \%$ \\
\hline 1 & Universidad del Pacífico & 3 & $2,4 \%$ \\
\hline 1 & Universidad ESAN & 3 & $2,4 \%$ \\
\hline 1 & Independiente & 2 & $1,6 \%$ \\
\hline 1 & Universidad Nacional Mayor de San Marcos & 2 & $1,6 \%$ \\
\hline 5 & $\begin{array}{l}\text { Otras instituciones peruanas con una participación como máximo en } \\
\text { publicaciones sobre el MILA }\end{array}$ & 5 & $4,0 \%$ \\
\hline 9 & Total de participaciones universidades peruanas & 15 & $11,9 \%$ \\
\hline 1 & Universidad de Valencia & 2 & $1,6 \%$ \\
\hline 1 & Universidad Politécnica de Valencia & 2 & $1,6 \%$ \\
\hline 2 & Total de participaciones universidades españolas & 4 & $3,2 \%$ \\
\hline 6 & $\begin{array}{l}\text { Instituciones de otros países con una participación como máximo en } \\
\text { publicaciones sobre el MILA }\end{array}$ & 6 & $4,8 \%$ \\
\hline 80 & Total general & 126 & $100 \%$ \\
\hline
\end{tabular}


Mercado Integrado Latinoamericano: diez años después de su creación. Un análisis bibliométrico para el periodo 20082021

En gran parte de las organizaciones patrocinadoras, resaltadas en la Tabla anterior, varios autores sobresalen por su amplio aporte investigativo a la literatura sobre el MILA. Según la Tabla 7, sólo 27 de los 152 investigadores que se tienen registrados (el 17,8\%) han contribuido en más de un manuscrito sobre este tema. Daniela Pérez Noreña, seguida por Belky Esperanza Gutiérrez Castañeda, ambas afiliadas a la Universidad de Antioquia, y por Luis Berggrun, patrocinado por la universidad Icesi, se destacan como los autores con el mayor número de publicaciones sobre el mercado integrado, exactamente con 5, 4 y 4 participaciones, respectivamente. Asimismo, con 3 artículos por cada uno sobresalen Edmundo Lizarzaburu Bolaños, por parte de la Universidad ESAN; Eduardo Sandoval Álamos, por las universidades de Concepción y Tecnológica Metropolitana de Chile; y Leovardo Mata, en representación del Tecnológico de Monterrey y la Universidad Autónoma Metropolitana de México.

Tabla 7. Principales autores que investigan sobre el mercado MILA

Table 7. Most prolific authors researching the MILA

\begin{tabular}{|c|c|c|}
\hline Autor & Número de participaciones & Afiliación institucional \\
\hline \multicolumn{3}{|l|}{ Por Colombia } \\
\hline Daniela Pérez Noreña & 5 & Universidad de Antioquia \\
\hline Belky E. Gutiérrez Castañeda & 4 & Universidad de Antioquia \\
\hline Luis Berggrun & 4 & Universidad Icesi \\
\hline Carlos Andrés Barrera Montoya & 2 & Universidad de Antioquia \\
\hline Diego Andrés Correa Mejía & 2 & Universidad de Antioquia \\
\hline Julián Esteban Zamarra Londoño & 2 & Universidad de Antioquia \\
\hline Felipe Alejandro Torres Castro & 2 & Universidad Militar Nueva Granada \\
\hline Jorge Armando Ortegón Rojas & 2 & Universidad Militar Nueva Granada \\
\hline Diana Milena Carmona Muñoz & 1 & Universidad Autónoma de Colombia \\
\hline$X X$ & 1 & Universidad Piloto de Colombia \\
\hline Diego A. Agudelo & 2 & Universidad EAFIT \\
\hline Jairo González Bueno & 2 & Universidad Pontificia Bolivariana \\
\hline José Rodrigo Vélez Molano & 2 & Universidad de La Salle \\
\hline \multicolumn{3}{|l|}{ Por Chile } \\
\hline \multirow[b]{2}{*}{ Eduardo Sandoval Álamos } & 2 & Universidad de Concepción \\
\hline & 1 & $\begin{array}{c}\text { Universidad Tecnológica } \\
\text { Metropolitana }\end{array}$ \\
\hline Carlos Leandro Delgado Fuentealba & 2 & Universidad de Concepción \\
\hline Jorge Andrés Muñoz Mendoza & 2 & Universidad de Concepción \\
\hline Sandra María Sepúlveda Yelpo & 2 & Universidad de Concepción \\
\hline Carmen Lissette Veloso Ramos & 2 & $\begin{array}{c}\text { Universidad de Concepción- } \\
\text { Universidad Santo Tomás }\end{array}$ \\
\hline \multicolumn{3}{|l|}{ Por España } \\
\hline Fernando García & 2 & Universidad Politécnica de Valencia \\
\hline Javier Oliver & 2 & Universidad Politécnica de Valencia \\
\hline \multicolumn{3}{|l|}{ Por México } \\
\hline Leovardo Mata & 2 & $\begin{array}{c}\text { EGADE Business School } \\
\text { (Tecnológico de Monterrey), con la } \\
\text { participación de la ECSG del }\end{array}$ \\
\hline
\end{tabular}




\begin{tabular}{|c|c|c|}
\hline & & $\begin{array}{l}\text { Tecnológico de Monterrey para una } \\
\text { de las publicaciones }\end{array}$ \\
\hline & 1 & $\begin{array}{c}\text { Universidad Autónoma } \\
\text { Metropolitana }\end{array}$ \\
\hline José Antonio Núñez Mora & 2 & $\begin{array}{c}\text { EGADE Business School } \\
\text { (Tecnológico de Monterrey) }\end{array}$ \\
\hline Roberto Joaquín Santillán Salgado & 2 & $\begin{array}{l}\text { EGADE Business School, Tec. de } \\
\text { Monterrey }\end{array}$ \\
\hline Ricardo Massa Roldán & 2 & $\begin{array}{c}\text { EGADE Business School (Tecnológico } \\
\text { de Monterrey y CONACYT-CIDE) }\end{array}$ \\
\hline Samuel Mongrut & 2 & $\begin{array}{c}\text { EGADE Business School } \\
\text { (Tecnológico de Monterrey y la } \\
\text { Universidad del Pacífico del Perú) }\end{array}$ \\
\hline Montserrat Reyna Miranda & 2 & $\begin{array}{l}\text { EGADE Business School (Tecnológico } \\
\text { de Monterrey con la participación } \\
\text { del FDDI (China) para una de las } \\
\text { publicaciones. }\end{array}$ \\
\hline \multicolumn{3}{|l|}{ Por Perú } \\
\hline Edmundo R. Lizarzaburu B. & 3 & Universidad ESAN \\
\hline \multirow[t]{2}{*}{ Kurt Burneo Farfán } & 1 & $\begin{array}{c}\text { Pontificia Universidad Católica del } \\
\text { Perú }\end{array}$ \\
\hline & 1 & Universidad San Ignacio de Loyola \\
\hline
\end{tabular}

Fuente: elaboración propia.

Seguidamente, el mapa de grafos que se presenta en la Figura 3, describe las redes de coautoría identificadas en los estudios publicados sobre el mercado MILA. La gama de colores permite distinguir las diferentes comunidades de trabajo. Cada autor fue codificado con una numeración y representado por un nodo (círculo). El tamaño de cada uno es proporcional a la cantidad de conexiones que tiene cada investigador con relación a otros. Asimismo, la solidez de las aristas que unen a los nodos indica el grado de proximidad y la fuerza de interacción entre ellos; es decir, el grosor de cada línea, que une a dos o más autores, se intensifica a medida en que estos participen de más artículos de manera conjunta.

En este orden de ideas, para el análisis de redes son incluidos los 73 documentos identificados en este estudio, de ellos, 61 son escritos por más de un autor, lo que equivale al 83,6\% del total. En términos de autores, de los 152 participantes, 140 presentan al menos una conexión con otro del listado, esto representa al 92,1\% de ellos. Ahora bien, analizando la Figura 3, es posible identificar un amplio aislamiento en los grupos de investigación que adelantan pesquisas sobre el mercado integrado. Según los estadísticos calculados por el software Gephi Versión 0.9.2, se identificaron 41 comunidades de trabajo, las cuales pueden distinguirse según la gama de colores, rasgo característico que da cuenta de que no existe un trabajo en equipo estratégicamente planeado y con alcance internacional. Sin embargo, en la periferia del grafo se han conglomerado aquellas comunidades con el mayor número de interacciones. De estas, cabe destacar al conjunto de nodos con color rosa, en el cual Luis Berggrun (cód. 26), de Colombia, seguido por Edmundo Lizarzaburu (cód. 45) y Kurt Burneo (cód. 70), ambos del Perú, aparte de sobresalir por su habitual trabajo en equipo (la solidez de sus aristas o líneas), se convierten en los autores con el mayor número de conexiones con otros investigadores, tanto dentro de su respectivo grupo como para toda la red de participantes del MILA. 
Luego, por número de interacciones con otros autores, también se destacan Samuel Mongrut (cód. 27), por parte de México-Perú, y Daniela Pérez Noreña (cód. 6) junto a Belky E. Gutierrez (cód. 4), ambas por Colombia. Estas últimas, además, debido a la solidez de su respectiva arista (línea que les une), se identifican como la dupla con el mayor número de apariciones en conjunto de toda la red, precisamente por sus recientes publicaciones y trabajo en equipo desde la Universidad de Antioquia. Otros investigadores que igualmente sobresalen en términos de interacciones dentro de su respectiva comunidad son Roberto Santillán-Salgado (cód. 22), en representación del EGADE Business School, y Eduardo Sandoval Álamos (cód. 30), por parte de la Universidad chilena de Concepción. De igual manera, la comunidad de origen chilena, formada por Jorge A. Muñoz (cód. 102), Sandra María Sepúlveda Yelpo (cód. 103), Carmen Lissette Veloso R. (cód. 104) y Carlos Leandro Delgado Fuentealba (cód. 105), y el conglomerado de autores de origen colombo-español, conformado por Fernando García (cód. 15), Jairo González B. (cód. 16) y Javier Oliver (cód. 17), si bien no prevalece en ellos un único protagonista sí que resultan relevantes, tanto en términos de conexiones como, por la cantidad de manuscritos que en conjunto han publicado entre los respectivos miembros de cada grupo.

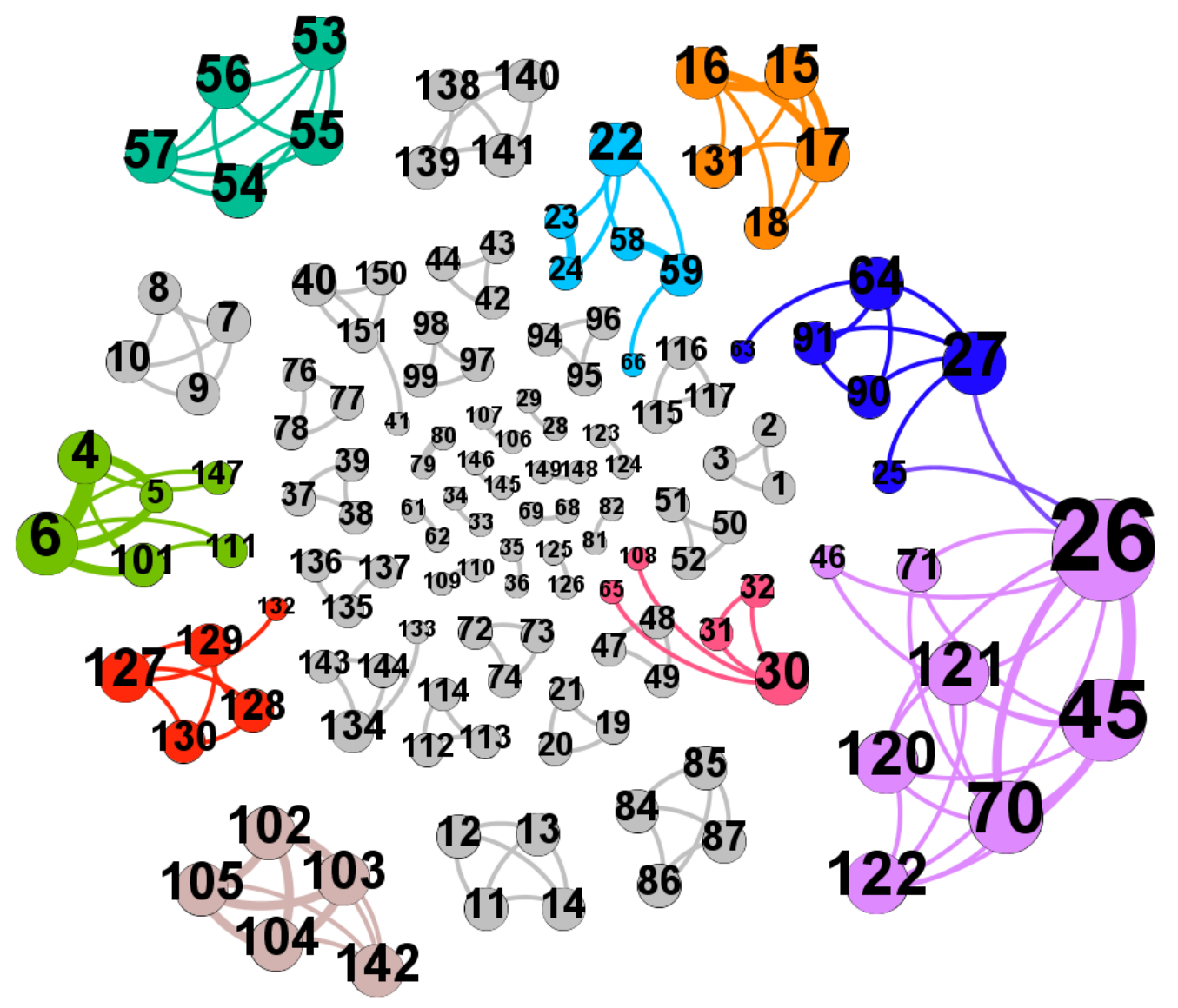

Figura 3. Red de coautoría en las investigaciones reportadas sobre el mercado MILA, periodo 2010-2021*

Figure 3. Co-authorship network of studies on the MILA (2010-2021)

Fuente: elaboración propia en el software Gephi V. 0.9.2 con base en la información reportada por Scopus, WoS y Google Académico. 
*Autores de las principales comunidades

\begin{tabular}{|c|c|c|c|}
\hline (4) Belky E. Gutiérrez & (30) Eduardo Sandoval Alamos & (64) Diego A. Agudelo & (111) Aura Pareja-Taborda \\
\hline (5) Carlos A. Barrera & (31) Arturo Z. Vásquez-Párraga & (65) Macarena Soto & $\begin{array}{c}\text { (120) Gabriela Barriga } \\
\text { Ampuero }\end{array}$ \\
\hline (6) Daniela Pérez N. & (32) Rocío Sabat A. & (66) Martha Mota A. & (121) Julio Cisneros \\
\hline (15) Fernando García & (45) Edmundo R Lizarzaburu Bolaños & (70) Kurt Burneo F. & (122) Luis E. Noriega \\
\hline (16) Jairo González B. & (46) Emilio Cardona & (71) Hamilton Galindo & $\begin{array}{c}\text { (127) José Rodrigo Vélez } \\
\text { Molano }\end{array}$ \\
\hline (17) Javier Oliver & (53) Nicolas Hardy & (90) Santiago E. Ibarraza & (128) María Barbosa \\
\hline (18) Rima Tamosiuniené & (54) Nicolas Magner & (91) Ma. Isabel Castro & (129) Milton Camelo \\
\hline (22) Roberto J. Santillán-Salgado & (55) Jaime Lavin & $\begin{array}{l}\text { (101) Julián E. Zamarra } \\
\text { Londoño }\end{array}$ & (130) Álvaro Pulido \\
\hline (23) Ricardo Massa R. & (56) Rodrigo A. Cárdenas & (102) Jorge A. Muñoz & (131) Francisco Guijarro \\
\hline (24) Montserrat Reyna Miranda & (57) Mauricio Jara-Bertin & $\begin{array}{l}\text { (103) Sandra María } \\
\text { Sepúlveda Yelpo }\end{array}$ & (132) Nicolás Morales León \\
\hline (25) Darcy Fuenzalida & (58) José A. Núñez M. & $\begin{array}{l}\text { (104) Carmen Lissette } \\
\text { Veloso R. }\end{array}$ & $\begin{array}{c}\text { (142) Rodrigo A. Fuentes- } \\
\text { Solís }\end{array}$ \\
\hline (26) Luis Berggrun & (59) Leovardo Mata & $\begin{array}{l}\text { (105) Carlos Leandro } \\
\text { Delgado Fuentealba }\end{array}$ & $\begin{array}{c}\text { (147) Daniel F. Giraldo } \\
\text { Osorio }\end{array}$ \\
\hline (27) Samuel Mongrut & (63) Luna Ramírez S. & (108) Fernando Olea & \\
\hline
\end{tabular}

Con relación al número de citaciones por documento, se advierte que, con corte a 31 de mayo de 2021, 27 de las 73 publicaciones seleccionadas, es decir, el 36,9\% no registran citación alguna en las bases de datos incluidas en este análisis, mientras que tan solo 13 (17,8\%) registran máximo una citación en al menos una de ellas. Los restantes 33 documentos $(45,2 \%)$ registran más de una citación en al menos una de las BD consideradas.

Para analizar la red de citaciones de la producción investigativa sobre el MILA, se consideró el número de citas que recibió cada uno de los artículos de la muestra, pero que hayan sido citados por el resto de artículos que conforma dicha muestra. Es decir, si alguno de los manuscritos de este estudio fue citado por un artículo que no forme parte de las 73 publicaciones que integran la muestra, dicha citación no se tendrá en cuenta. Luego de la anterior depuración se evidencia, en principio, la baja conexión entre artículos relacionados estrictamente con la temática del mercado integrado. Uno de los casos que podría servir de ilustración sobre la poca conexión entre las nuevas producciones sobre el MILA y la literatura previa que aborda esta misma línea es el manuscrito con código 29, escrito por Lizarzaburu Bolaños et al. (2015), el cual ha recibido un total de 41, 14 y 9 citaciones según Google académico, Scopus y WoS, respectivamente, pero si el análisis se restringe a saber cuántas de esas citas provienen desde (corresponden a) los artículos que integran la muestra, se confirma que dicho manuscrito ha recibido tan solo 7,3 y 3 citas, respectivamente.

La matriz de citaciones así depurada fue representada mediante un mapa de grafos en la Figura 4. En este, la gama de colores describe las comunidades de citaciones. Cada manuscrito fue codificado con un número y representado por un nodo (círculo), su tamaño es proporcional al número de citaciones que recibe (indegree). Las líneas son unidireccionales representadas con una flecha, su punto de llegada (cabeza) se refiere al manuscrito que ha recibido alguna citación, su punto de partida (cola) proviene del manuscrito que ha emitido la cita, ejemplo, el manuscrito 18 ha sido citado por el 31. En este sentido, de los 73 documentos incluidos en el análisis, 48 (65.8\%) lograron hacerse a un nodo dentro del grafo, y es que el requisito para tener representación dentro del mapa consiste en ser 
Mercado Integrado Latinoamericano: diez años después de su creación. Un análisis bibliométrico para el periodo 20082021

citado por/citar a uno de los documentos de la muestra, como mínimo. Nuevamente queda en evidencia los bajos niveles de conexión entre las publicaciones relacionadas con el MILA.

Ahora bien, tras el análisis de la Figura 4, pueden identificarse ocho comunidades de citación según la gama de colores en que se encuentra distribuido el grafo. En ellas sobresalen varios nodos (documentos) por su tamaño de acuerdo con la cantidad de flechas que recibe (número de citaciones). Según el grafo, 9 es el máximo de citas que ha recibido una publicación sobre el MILA por otras publicaciones estrictamente relacionadas con dicha temática. Este es el caso del documento con código 28, escrito por Vargas Pulido y Bayardo Martínez (2013), el cual, al abordar de forma reflexiva (artículo no empírico) varias ventajas y desafíos alrededor del acuerdo, se convirtió en el referente con el mayor número de citas hasta la fecha, tanto en su comunidad como en la nueva literatura que se ha venido forjando alrededor de esta integración. A la par con el anterior artículo y con 7 citaciones recibidas por cada uno, se destacan los documentos con código 29, 25 y 18 dentro de la comunidad color rosa, escritos por Lizarzaburu Bolaños et al. (2015), Sandoval y Soto (2016) y Romero-Álvarez et al. (2013) (cód. 18), respectivamente, siendo también referente dentro de este tipo de literatura. De igual forma, con 6 citaciones recibidas, el manuscrito con el código 11, escrito por Mellado y Escobari (2015), sobresale como referente dentro de su respectiva comunidad.

Respecto a los aspectos metodológicos y temáticas abordadas por estos referentes, se resalta que, en el caso de Lizarzaburu Bolaños et al. (2015), utilizan un análisis estadístico básico al evaluar el impacto del MILA en diversos indicadores del mercado bursátil, entre ellos los niveles de correlación, mientras que en Sandoval y Soto (2016), Romero-Álvarez et al. (2013) y Mellado y Escobari (2015), por el contrario, se prefiere seleccionar modelos cuantitativos mucho más avanzados, como el test de Johansen, la técnica de análisis por componentes y el modelo de correlaciones propuesto por Engle (2002), respectivamente, y a pesar de la diversidad en las metodologías escogidas, estos tres últimos estudios evidenciaron un efecto integración entre los mercados que inicialmente conformaron el acuerdo, aludiendo así, a una posible disminución en los beneficios de diversificación de portafolios para el inversor.

A partir de los referentes mencionados, en especial de aquellos pertenecientes a la comunidad de color rosa, la temática sobre la integración financiera entre los mercados miembros comenzó a tomar protagonismo en publicaciones posteriores, hasta el punto de convertirse en el tema de moda dentro de la literatura del MILA. Evidencia de ello son los estudios publicados por Rojas-Mora y ChamorroFutinico (2016) (cód. 9), Álamos y Rodríguez (2020) (cód. 50), quienes utilizan nuevamente el test de Johansen en su trabajo empírico, o las pesquisas dirigidas por Espinosa-Méndez et al. (2017) (cód. 17), Mota Aragón y Mata Mata (2017) (cód. 26), Reyes Zárate (2016) (cód. 27) y Muñoz Mendoza et al. (2020) (cód. 48), en las que se evidencia el uso de una variedad de modelos pertenecientes a la familia Garch; del mismo modo, las publicaciones de Ortegón y Torres (2016) (cód. 34) y Mata y Núñez Mora (2016) (cód. 31), que constatan la codependencia entre los rendimientos del MILA respecto a mercados foráneos como Latibex o el mismo mercado financiero de China, respectivamente, aportan más evidencia sobre el amplio desarrollo de esta temática.

Con niveles mucho más bajos de citación, y aunque se encuentren prácticamente al margen de los estudios referentes, se han reportado otras publicaciones que persisten en aportar más evidencia sobre la integración financiera entre los mercados del acuerdo utilizando diversas metodologías. Este es el caso de Duarte Duarte et al. (2019) (cód. 4) y Santillán-Salgado et al. (2017) (cód. 7), 
caracterizados por emplear un análisis de correlación y causalidad, o el presentado por Castro y Marín (2014) (cód. 22), que se distingue por seleccionar un modelo de correlaciones condicionales constantes, propuesto por Engle (2002).

Por otra parte, ota de las líneas de investigación que aperturaron los estudios referentes, pero que aún se encuentra en etapa de ascenso, es la de contrastar la HME dentro del MILA. Las tres formas de eficiencia reconocidas por la literatura financiera ya han sido probadas en los mercados de la integración a través de diversas metodologías. En su forma más débil, Arbeláez García y Rosso (2016) (cód. 49) verificaron la HME al indagar por la existencia de ciertas anomalías de calendario (estacionales) en la serie de los retornos de los mercados miembros. Bajo su formato semifuerte, los estudios relacionados prefirieron por verificar parcialmente esta teoría a través de la habilidad con que el retorno accionario incorpora la información relevante de una variable en particular, es el caso de la información contenida en los anuncios de ganancias (Hardy et al., 2018) (cód. 19) o en el dictamen de auditoría (Gutiérrez Castañeda et al., 2020a) (cód. 51). Finalmente, en contraste con los anteriores estudios, Ruiz Dávila y García Muñoz (2020) (cód. 52) prefirieron por confirmar los tres tipos de eficiencia de mercado basándose en la teoría de caminata aleatoria y verificando si los principales índices de los participantes del MILA se comportan bajo alguna de las tipologías de caminata propuesta por Campbell et al. (1997).

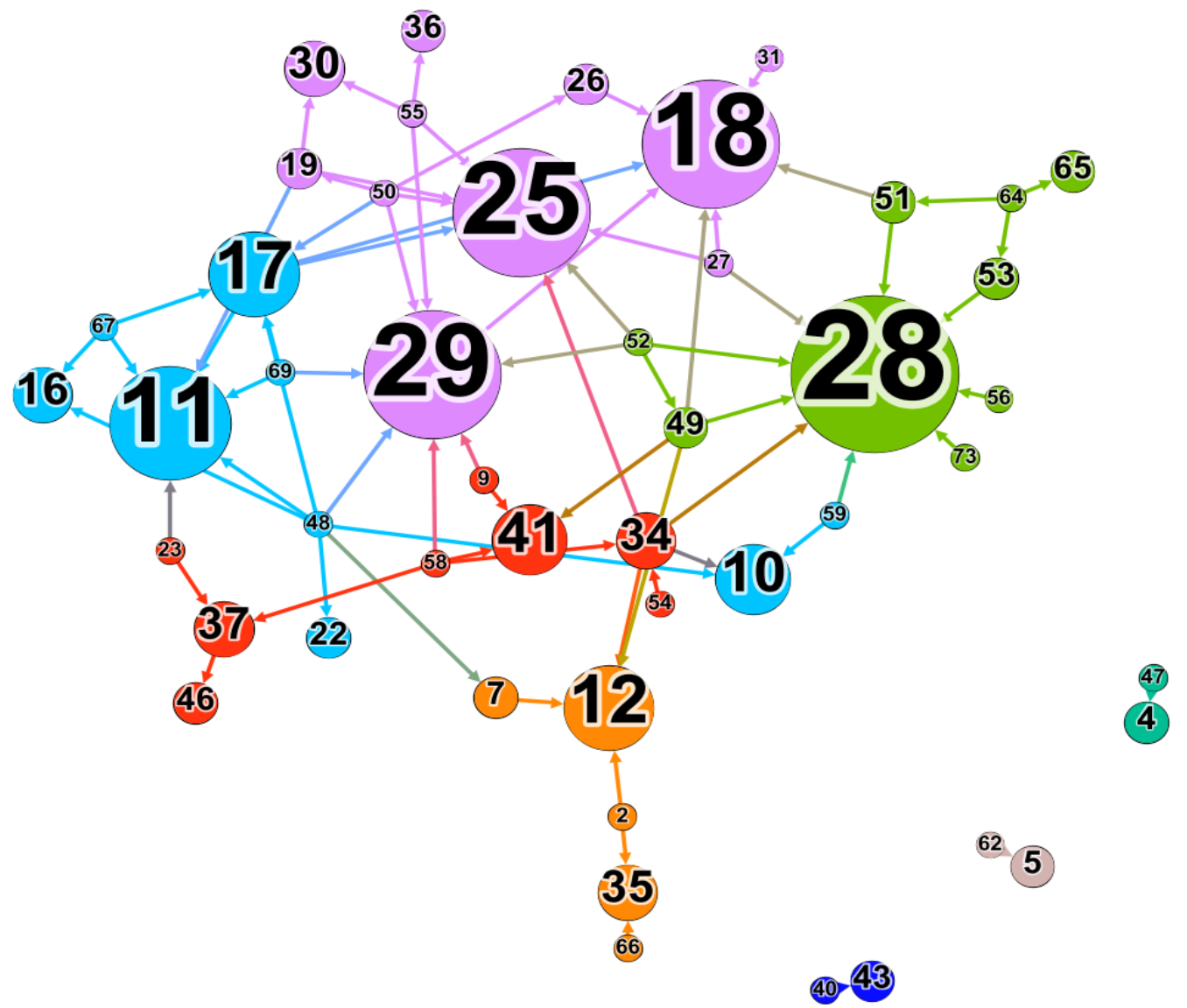

Figura 4. Red de citaciones recibidas por documento con corte a 31 de mayo de $2021 *$

Figure 4. Network of citations per document (until May 31, 2021)

Fuente: elaboración propia en el software Gephi V. 0.9.2 con base en la información reportada por Scopus, WoS y Google Académico. 
Mercado Integrado Latinoamericano: diez años después de su creación. Un análisis bibliométrico para el periodo 20082021

* Referencias con más citas

\begin{tabular}{|c|c|c|c|}
\hline Cód.) Documento & Cód.) Documento & Cód.) Documento & Cód.) Documento \\
\hline (4) Duarte Duarte et al. (2019) & $\begin{array}{c}\text { (18) Romero-Álvarez et al. } \\
\text { (2013) }\end{array}$ & $\begin{array}{l}\text { (29) Lizarzaburu Bolaños et al. } \\
\text { (2015) }\end{array}$ & $\begin{array}{c}\text { (51) Gutiérrez Castañeda et } \\
\text { al. (2020a) }\end{array}$ \\
\hline (7) Santillán-Salgado et al. (2017) & (19) Hardy et al. (2018) & (31) Mata y Núñez Mora (2016) & $\begin{array}{c}\text { (52) Ruiz Dávila y García } \\
\text { Muñoz (2020) }\end{array}$ \\
\hline $\begin{array}{c}\text { (9) Rojas-Mora y Chamorro- } \\
\text { Futinico (2016) }\end{array}$ & (22) Castro y Marín (2014) & (34) Ortegón y Torres (2016) & $\begin{array}{l}\text { (55) Galicia-Anaya et al. } \\
\text { (2018) }\end{array}$ \\
\hline (10) Sandoval Álamos et al. (2015) & (25) Sandoval y Soto (2016) & (41) Agudelo et al. (2012) & (58) Farfán et al. (2017) \\
\hline (11) Mellado y Escobari (2015) & $\begin{array}{c}\text { (26) Mota Aragón y Mata } \\
\text { Mata (2017) }\end{array}$ & $\begin{array}{l}\text { (48) Muñoz Mendoza et al. } \\
\text { (2020) }\end{array}$ & $\begin{array}{c}\text { (64) Torres-Cano y Correa- } \\
\text { Mejía (2021) }\end{array}$ \\
\hline $\begin{array}{l}\text { (12) Uribe Gil y Mosquera López } \\
\text { (2014) }\end{array}$ & (27) Reyes Zárate (2016) & $\begin{array}{c}\text { (49) Arbeláez García y Rosso } \\
\text { (2016) }\end{array}$ & $\begin{array}{c}\text { (67) Muñoz Mendoza et al. } \\
\text { (2021) }\end{array}$ \\
\hline $\begin{array}{c}\text { (17) Espinosa-Méndez et al. } \\
(2017)\end{array}$ & $\begin{array}{c}\text { (28) Vargas Pulido y Bayardo } \\
\text { Martínez (2013) }\end{array}$ & (50) Álamos y Rodríguez (2020) & $\begin{array}{l}\text { (69) Garzón Jiménez y Zorio- } \\
\text { Grima (2021) }\end{array}$ \\
\hline
\end{tabular}

\section{DISCUSIÓN}

Este estudio presentó un análisis bibliométrico de las publicaciones relacionadas con el Mercado Integrado Latinoamericano. En términos agregados, si bien los manuscritos alrededor de este tema se han mantenido casi de forma ininterrumpida durante los años que lleva de funcionamiento, con presencia en revistas tipo Scopus y/o WoS, existe una significativa dispersión en el desarrollo investigativo alrededor de la infraestructura MILA cuando se soporta un análisis en revista, institución, autor, coautoría y citación.

Aunque se ha subrayado que a la fecha no se tiene conocimiento sobre un estudio similar a este que realice a profundidad un análisis bibliométrico sobre las publicaciones en torno al mercado MILA, sí que podría tomarse como posible referente de comparación el artículo presentado por Ortegón Rojas y Torres Castro (2016), en el cual se realiza una revisión de la literatura respecto a esta integración. En este sentido, den relación con los resultados obtenidos, más allá de enumerar las metodologías utilizadas por los artículos sobre el MILA -según lo documentó la pesquisa de referencia-, en este estudio se presentan las relaciones existentes entre ellos que permitan, a su vez, identificar cuáles de estos son los referentes teóricos, así como los autores, instituciones, países y revistas que se destacan en este tipo de literatura.

Como valor agregado, y en busca de atacar el aislamiento identificado entre los estudios relacionados por Ortegón Rojas y Torres Castro (2016), esta investigación clasifica los artículos por grandes temáticas, identificando las de mayor desarrollo y conexión alrededor del acuerdo, como lo son las líneas sobre integración financiera y contraste de la HME; las que con un moderado desarrollo presentan poca conexión entre sus publicaciones, este es el caso de los tópicos sobre estructuración eficiente de portafolios, reportes integrados y factores determinantes de los retornos en el MILA; Y las temáticas que, a pesar de ser relevantes para la eficiencia transaccional del mercado, evidencian un escaso desarrollo investigativo, entre ellas se destacan los factores de liquidez, tipo de cambio y reglas fiscales al interior de la integración.

Así mismo, al clasificarse las publicaciones en grandes temáticas, este artículo contribuirá para que a futuro nuevas investigaciones puedan proseguir y profundizar de manera empírica en las líneas ya mencionadas y que hasta ahora son consideradas incipientes en el campo investigativo, pero de gran peso en la operatividad del mercado, o en aquellas que, por el contrario, reflejan un moderado 
avance, aunque con un pronunciado aislamiento entre sus publicaciones. Se espera que la literatura relacionada y caracterizada en este estudio pueda servir de referente teórico dentro de los nuevos aportes científicos que se tejerán alrededor de las integraciones para la nueva década que comienza.

Por otra parte, las limitaciones de este estudio hacen referencia a los bajos niveles de producción científica sobre el mercado integrado durante casi una década de funcionamiento, por lo que condicionó un análisis mucho más profundo y especializado. Otra de las limitaciones presente, y que podría ser abordada en futuras investigaciones, es que la búsqueda y selección de artículos en esta pesquisa se delimitó a aquellas publicaciones que en sus respectivos títulos, resúmenes o palabras clave, evidenciaran el término MILA, Mercado Integrado Latinoamericano, Latin American Integrated Market $u$ otras palabras similares. Este procedimiento probablemente excluyó una serie de manuscritos que también le aportaron a la integración, ya sea de un modo individual al considerar en sus análisis uno o dos países miembros del acuerdo, o tal vez, por desconocer el nombre dado a este mercado bursátil, utilizaron el término Alianza del Pacífico, países andinos o incluso Latinoamérica para referirse a los tres o cuatro países miembros de la integración, según el rango de tiempo escogido para ser analizado.

A futuro, según el reconocimiento que se haga a los trabajos previos en forma de citaciones, acompañado de un mayor dinamismo y cohesión entre las redes de autores y comunidades de trabajo identificadas en este análisis, con alcances y objetivos estratégicos establecidos regionalmente, dependerá que la investigación sobre el MILA se torne mucho más sólida, inclusiva y visible. Al mismo tiempo, por la evidencia que logren aportar las nuevas investigaciones que se propongan, sería de relevancia para los participantes y reguladores actuales del mercado y para aquellos que se pretenden adherir en un futuro cercano a la AP, y, por ende, al MILA, en pro de mayor desarrollo y madurez del mismo Mercado Integrado Latinoamericano.

\section{CONCLUSIONES}

La puesta en marcha del MILA entre los participantes bursátiles de Chile, Colombia y Perú, con la posterior incorporación de México, ha motivado el desarrollo de toda una literatura que hasta ahora se considera emergente. A diez años de su creación, solo se identificaron 73 publicaciones entre los años 2008 y 2021 en las bases de datos Scopus, WoS y Google Académico. Estos manuscritos permitieron analizar las características de la producción científica y la evolución de las temáticas que se han adelantado en torno a este acuerdo. Para ello fue aplicado un estudio bibliométrico basado principalmente en técnicas descriptivas en compañía de un análisis de gráficos y de redes de citaciones y coautorías a través de la utilización de grafos.

Los resultados permiten concluir que, a pesar de la producción sostenida alrededor de la media de publicaciones por año para el periodo 2015-2020, del creciente interés investigativo tanto por organizaciones y autores de la región como por fuera de ella, así como el espacio que ha ido ganando en las discusiones internacionales por las publicaciones sobre el MILA en revistas tipo Scopus y/o WoS, existe todavía una marcada tendencia por publicar en revistas de la región latina, principalmente en medios colombianos, los cuales, en su mayoría, se caracterizan por no estar indexados en las anteriores bases de datos. 
Adicional a esto, si bien se destacan en sus respectivas categorías a la Universidad de Antioquia, a Colombia como conglomerado de instituciones, a Daniela Pérez Noreña/Luis Berggrun como autor principal por número de publicaciones/de conexiones y al artículo referente publicado por Vargas Pulido y Bayardo Martínez (2013), es evidente que la falta de especialidad en estos temas del MILA por parte de las revistas, instituciones, autores y comunidades de trabajo, además del bajo número de citaciones que han recibido las publicaciones sobre el MILA por otras publicaciones estrictamente relacionadas con dicha alianza, dan cuenta de un escaso trabajo planificado en la región y una marcada fragmentación en el desarrollo investigativo alrededor de este tema, especialmente en lo que tiene que ver con aquellas aristas relevantes para la madurez y progreso del acuerdo, diferentes a la integración financiera y a la comprobación de la HME.

\section{CONFLICTOS DE INTERÉS}

El autor declara que no presenta conflictos de interés financiero, profesional o personal que pueda influir de forma inapropiada en los resultados obtenidos o las interpretaciones propuestas.

\section{REFERENCIAS}

Acevedo Arango, H. D., Becerra Arevalo, J. A., Guisao Usuga, F. D. (2020). Corporate governance and probability of improve Tobins Q: A study case in Latin America. Revista Espacios, v. 41, n. 14, 4. $\underline{U R L}$

Agudelo, D. A., Barraza, S. E., Castro, M. I., Mongrut, S. (2012). Liquidez en los mercados accionarios latinoamericanos: Estimando el efecto del Mercado Integrado Latinoamericano (MILA). Centro de Investigación en Economía y Finanzas (CIEF), Documentos de trabajo No. 12-21. https://doi.org/10.2139/ssrn.2400734

Álamos, E. S., Rodríguez, F. O. (2020). Latin American integrated market: is there cointegration after the entry of Mexico? GCG: Revista de Globalización, Competitividad y Gobernabilidad, v. 14, n. 3, 34-47. URL

Álvarez-Flores, L., Cortez-Alejandro, K. A., Rositas-Martínez, J. (2020). Efecto de la equidad como dimensión de calidad de vida laboral en el desempeño financiero del Mercado Integrado Latinoamericano. VinculaTégica EFAN, v. 6, n. 1, 382-391. URL

Améstica Rivas, L., King Domínguez, A., Moraga Dionicio, V., Romero Romero, R. (2021). Influencia del directorio en el desempeño del Mercado Integrado Latinoamericano. Revista Finanzas y Política Económica, v. 13, n. 1, 225-251. https://doi.org/10.14718/revfinanzpolitecon.v13.n1.2021.10

Arbeláez García, D., Rosso, J. (2016). Efectos estacionales en los mercados de capitales de la Alianza del Pacífico. Estudios Gerenciales, v. 32, n. 141, 358-368.

http://doi.org/10.1016/i.estger.2016.10.002

Ardanuy, J. (2012). Breve introducción a la bibliometría. Universitat de Barcelona. URL 
Arduini, D., Zanfei, A. (2014). An overview of scholarly research on public e-services? A meta-analysis of the literature. Telecommunications Policy, v. 38, n. 5-6, 476-495.

https://doi.org/10.1016/j.telpol.2013.10.007

Arias-Ciro, J. (2020). Estudio bibliométrico de la eficiencia del gasto público en educación. Revista CEA, v. 6, n. 11, 127-144. https://doi.org/10.22430/24223182.1588

Ariza, M., Castillo, S., Córdoba, F., Velosa, F. (2012). MILA: la oportunidad para invertir en un ETF. Sinapsis, v. 4, n. 4, 69-89. URL

Baquero-Herrera, M. (2013). Legal certainty and financial markets integration: The MILA case. Law and Business Review of the Americas, v. 19, n. 4, 487-502. URL

Basnet, H. C., Sharma, S. C., Vatsa, P. (2015). Monetary policy synchronization in the ASEAN-5 region: an exchange rate perspective. Applied Economics, v. 47, n. 1, 100-112. https://doi.org/10.1080/00036846.2014.962228

Berggrun, L., Lizarzaburu, E., Cardona, E. (2016). Idiosyncratic volatility and stock returns: Evidence from the MILA. Research in International Business and Finance, v. 37, 422-434. https://doi.org/10.1016/j.ribaf.2016.01.011

Campbell, J., Lo, A., MacKinlay, C. (1997). The Econometrics of Financial Markets. Princeton University Press. https://doi.org/10.2307/j.ctt7skm5

Carmona Muñoz, D. M., Vera Leyton, M. (2017). Evaluación de los factores de riesgo en los activos de renta variable que conforman el índice S\&P MILA 40: aplicación del modelo de tres factores de Fama y French en el periodo 2009-2013. Revista Finanzas y Política Económica, v. 9, n. 2, 301-317. https://doi.org/10.14718/revfinanzpolitecon.2017.9.2.5

Carvalho, M. M., Fleury, A., Lopes, A. P. (2013). An overview of the literature on technology roadmapping (TRM): Contributions and trends. Technological Forecasting and Social Change, v. 80, n. 7, 1418-1437. https://doi.org/10.1016/i.techfore.2012.11.008

Castro, C., Marín, N. J. (2014). Stock return comovements and integration within the Latin American integrated market (Working Paper No. 154). Universidad del Rosario.

Chávez Muñoz, N. M., Ríos Hernández, H. F., Carmona Muñoz, D. M. (2020). Determinantes macroeconómicos de la valoración de acciones: Caso alianza del pacífico. Dimensión Empresarial, v. 18, n. 1, 129-146. https://doi.org/10.15665/dem.v18i(1).2066

Coleman, S., Leone, V., de Medeiros, O. R. (2019). Latin American stock market dynamics and comovement. International Journal of Finance \& Economics, v. 24, n. 3, 1109-1129. https://doi.org/10.1002/ijfe.1708 
Dicaprio, A., Santos-Paulino, A. U. (2011). Can free trade agreements reduce economic vulnerability? South African Journal of Economics, v. 79, n. 4, 350-375.

https://doi.org/10.1111/j.1813-6982.2011.01310.x

Duarte Duarte, J., Garcés Carreño, L., Vargas Ayala, S., Vásquez González, V. (2019). Evaluación de la integración financiera entre los países pertenecientes al Mercado Integrado Latinoamericano. En F. Garrigós Simón; S. Estellés Miguel; I. Lengua Lengua; J. Onofre Montesa; C. M. Dema Pérez; J. V. Oltra Gutiérrez; Y. Narangajavana; M. J. Verdecho Sáez. (Eds.), International Conference On Innovation, Documentation and Education-INNODOCT/18 (399-411). Editorial Universitat Politècnica de València. http://dx.doi.org/10.4995/INN2018.2018.8785

Duarte Herrera, L. K., González Parías, C. H., Montoya Uribe, D. A. (2014). Colombia de Cara al nuevo regionalismo renovado: la Alianza del Pacífico. Revista Punto de Vista, v. 5, n. 9, 137-162. https://doi.org/10.15765/pdv.v5i9.568

Durieux, V., Gevenois, P. A. (2010). Bibliometric Indicators: Quality Measurements of Scientific Publication. Radiology, v. 255, n. 2, 342-351. https://doi.org/10.1148/radiol.09090626

Durisin, B., Calabretta, G., Parmeggiani, V. (2010). The intellectual structure of product innovation research: a bibliometric study of the journal of product innovation management, 19842004. Journal of Product Innovation Management, v. 27, n. 3, 437-451. https://doi.org/10.1111/j.1540-5885.2010.00726.x

Engle, R. (2002). Dynamic Conditional Correlation. Journal of Business \& Economic Statistics, v. 20, n. 3, 339-350. https://doi.org/10.1198/073500102288618487

Espinosa-Méndez, C., Gorigoitía, J., Vieito, J. (2017). Is the Virtual Integration of Financial Markets Beneficial in Emerging Markets? Evidence from MILA. Emerging Markets Finance and Trade, v. 53, n. 10, 2279-2302. https://doi.org/10.1080/1540496X.2017.1307101

Farfán, K. B., Lizarzaburu, E. R., Ampuero G. B., Preciado, L. B., Cisneros, J., Noriega Febres, L. E. (2017). Stock exchange in emerging markets: Integrated Latin America Market (MILA) - an opportunity for investment. Corporate Ownership \& Control, v. 14, n. 4-1, 227-239. https://doi.org/10.22495/cocv14i4c1art5

Fuenzalida, D., Berggrun, L., Mongrut, S. (2017). Illiquidity Premium in the MILA. Emerging Markets Finance and Trade, v. 53, n. 5, 1015-1029. https://doi.org/10.1080/1540496X.2016.1220858

Galicia-Anaya, S. A.; Rivas-Tovar, L. A.; Cárdenas Tapia, M. (2018). Las bolsas de valores en los países de la Alianza del Pacifico. En J. Sánchez-Gutiérrez; A. Vizcaíno; J. Gaytán-Cortés. (Coords.), Estrategias Financieras y Económicas promotoras de la Competitividad de las Organizaciones (122-151). Editorial Universidad de Guadalajara. 
Gallardo Loaiza, J. A. (2014). "Gobiernos corporativos": comparación entre Chile, Perú y Colombia como socios del Mercado Integrado Latinoamericano (MILA). Revista de Investigación Aplicada en Ciencias Empresariales, v. 3, n. 1, 7-29.

https://doi.org/10.22370/riace.2014.3.1.1819

García, F., González-Bueno, J., Guijarro, F., Oliver, J. (2020). A multiobjective credibilistic portfolio selection model. Empirical study in the Latin American integrated market. Entrepreneurship and Sustainability Issues, v. 8, n. 2, 1027-1046. http://doi.org/10.9770/jesi.2020.8.2(62)

García, F., González-Bueno, J., Oliver, J., Tamošiūnienè, R. (2019). A credibilistic mean-semivariancePER portfolio selection model for Latin America. Journal of Business Economics and Management, v. 20, n. 2, 225-243. https://doi.org/10.3846/ibem.2019.8317

Garzón Jiménez, R. G., Zorio-Grima, A. (2021). Sustainability engagement in Latin America firms and cost of equity. Academia Revista Latinoamericana de Administración, v. 34, n. 2. https://doi.org/10.1108/ARLA-05-2020-0117

Gil-Solano, D. (2020). Análisis del marco tributario de los países miembros del MILA y su efecto en la negociación de acciones entre países. Revista Punto de vista, v. 12, n. 17, 30-48. https://doi.org/10.15765/pdv.v12i17.1668

Giraldo López, J. A., Soto Sossa, M., Mejía Delgado, J. A. (2018). Características de la información contenida en los reportes integrados: una visión para el mercado integrado de américa latina (MILA). Contaduría Universidad de Antioquia, n. 73, 163-198. http://doi.org/10.17533/udea.rc.n73a07

Gutiérrez Betancur, J. C., Gutiérrez Díaz, A. K., Gómez Fernández, A. (2017). Estimación robusta de betas y el ratio de cobertura sobre futuros de índices bursátiles en el Mercado Integrado Latinoamericano (MILA). Ecos de Economía: A Latin American Journal of Applied Economics, v. 21, n. 44, 37-71. https://doi.org/10.17230/ecos.2017.44.2

Gutiérrez Castañeda, B. E., Barrera Montoya, C. A., Pérez Noreña, D. (2020a). Informe de auditoría y su relación con el mercado integrado latinoamericano (MILA). Apuntes Contables, n. 26, 101126. https://doi.org/10.18601/16577175.n26.07

Gutiérrez Castañeda, B. E., Barrera Montoya, C. A., Pérez Noreña, D. (2020b). Relationship Between Systematic and Idiosincratic Risk with the Expected Returns of Mila and Bm \& Fbovespa. International Journal of Business Marketing and Management, v. 5, n. 5, 12-25. URL

Hardy, N., Magner, N. S., Lavin, J., Cárdenas, R. A., Jara-Bertin, M. (2018). Small consequences of a major agreement: the MILA case. Academia Revista Latinoamericana de Administración, v. 31, n. 3, 486-518. https://doi.org/10.1108/ARLA-12-2017-0357

Hennings Otoya, J. A. (2015). Tributación y la cultura empresarial en la competencia del Mercado Integrado Latinoamericano. Quipukamayoc, v. 23, n. 44, 27- 34. https://doi.org/10.15381/quipu.v23i44.11623 
Hernández-Gamarra, K., Sarmiento-Sabogal, J., Cayon-Fallon, E. (2015). A test of the market efficiency of the integrated Latin American market (MILA) index in relation to changes in the price of oil. International Journal of Energy Economics and Policy, v. 5, n. 2, 534-539. URL

Hurtado Briceño, A. J. (2017). Avances en la Integración Financiera de la Alianza del Pacífico. En J. U. Mora Mora; M. I. Osorio-Caballero. (Coords.), La Alianza del Pacífico: Nuevos retos e implicaciones para Latinoamérica (125-142). Sello Editorial Javeriano.

Idrogo, W. (2019). Eficiencia del costo de oportunidad de capital usando betas para empresas y rendimientos del mercado integrado latinoamericano-MILA 2014-2017. Tzhoecoen, v. 11, n. 1, 42-56. https://doi.org/10.26495/rtzh1911.130006

Jaén, M. H., Auletta, N., Bruni Celli, J., Pocaterra, M. (2018). Bibliometric analysis of indexed research on corporate social responsibility in Latin America (2000-2017). Academia Revista Latinoamericana de Administración, v. 31, n. 1, 105-135.

https://doi.org/10.1108/ARLA-06-2017-0190

Jaramillo Arango, M., Ríos Restrepo, W. A., Correa-Mejía, D. A. (2020). Incidencia de factores ambientales y sociales en la generación de valor. Revista Facultad de Ciencias Económicas, v. 28, n. 2, 49-65. https://doi.org/10.18359/rfce.4033

King, A., Llinàs-Audet, J., Améstica Rivas, L. (2019). Gobiernos corporativos en universidades: Un estudio bibliométrico. Revista de Ciencias Sociales, v. 25, n. 1, 111-129. URL

Leraul, D. J. (2016). Trading with Neighbors: Regional Stock Exchange Integration-The Mercado Integrado Latinoamericano. Latin American Business Review, v. 17, n. 1, 49-71. https://doi.org/10.1080/10978526.2015.1137478

Lizarzaburu Bolaños, E. R., Burneo, K., Galindo, H., Berggrun, L. (2015). Emerging markets integration in Latin America (MILA) stock market indicators: Chile, Colombia, and Peru. Journal of Economics, Finance and Administrative Science, v. 20, n. 39, 74-83. https://doi.org/10.1016/i.jefas.2015.08.002

Luna-Ramírez, S., Agudelo, D. A. (2019). ¿Agrega Valor el Modelo Black-Litterman en Portafolios del Mercado Integrado Latinoamericano (MILA)? Evaluación Empírica 2008-2016. Revista de Métodos Cuantitativos para la Economía y la Empresa, v. 27, 55-73. http://dx.doi.org/10.2139/ssrn.3297111

Maruy, C., Aroca, F., Torretti, E., Villaseñor-Tadeo, G. (2015). Incidencia Tributaria del Mercado Integrado Latinoamericano: Alcances y Propuestas. IUS ET VERITAS, v. 24, n. 50, 408-422. URL

Mata, L., Núñez Mora, J. A. (2016). Dependence between the Chinese and MILA stock markets. Journal of Chinese Economic and Foreign Trade Studies, v. 9, n. 3, 234-244. http://doi.org/10.1108/JCEFTS-10-2016-034 
Mellado, C., Escobari, D. (2015). Virtual integration of financial markets: a dynamic correlation analysis of the creation of the Latin American Integrated Market. Applied Economics, v. 47, n. 19, 1956-1971. http://doi.org/10.1080/00036846.2014.1002892

Meneses Cerón, L. Á., Pérez Pacheco, C. A. (2020). Análisis comparativo de eficiencia en mercados emergentes. El caso de Colombia, Chile y Perú. Apuntes Contables, n. 26, 9-24. https://doi.org/10.18601/16577175.n26.02

Morales León, N., Vélez Molano, J. R. (2020). Cambios estructurales en índices bursátiles del mercado MILA entre los años 2008 y 2018. Semestre Económico, v. 23, n. 54, 21-44. https://doi.org/10.22395/seec.v23n54a2

Mota Aragón, M. B., Mata Mata, L. (2017). Volatilidad del Mercado Integrado Latinoamericano: un enfoque multivariado. Estocástica: Finanzas y Riesgo, v. 7, n. 1, 9-26. URL

Muñoz Mendoza, J. A., Sepúlveda Yelpo, S. M., Veloso Ramos, C. L., Delgado Fuentealba, C. L. (2020). Effects of MILA on their stock markets: an empirical analysis on market activity and dynamic correlations. International Journal of Emerging Markets, v. ahead-of-print, n. ahead-of-print. https://doi.org/10.1108/IJOEM-12-2019-1070

Muñoz Mendoza, J. A., Veloso Ramos, C. L., Sepúlveda Yelpo, S. M., Delgado Fuentealba, C. L., Fuentes-Solís, R. A. (2021). Impact of earnings management on agency costs: evidence from MILA markets. Baltic Journal of Management, v. 16, n. 2, 247-275. https://doi.org/10.1108/BJM-04-2020-0112

Núñez-Mora, J. A., Santillán-Salgado, R. J., Mata, L. (2017). Efficient portfolios and the generalized hyperbolic distribution. Economics Bulletin, v. 37, n. 4, 2711-2727. URL

Orozco Álvarez, J. E., Ramírez López, B. D. (2016). Análisis comparativo de los mercados bursátiles que integran el MILA. Contexto, v. 5, 53-62. https://doi.org/10.18634/ctxj.5v.0i.382

Ortegón Rojas, J., Torres Castro, F. (2016). El mercado integrado latinoamericano MILA, estado del arte. Cuadernos Latinoamericanos de Administración, v. 12, n. 23, 7-15. https://doi.org/10.18270/cuaderlam.v12i23.2111

Ortegón, J., Torres, F. (2016). El Mercado Integrado Latinoamericano (MILA): sus expectativas y el impacto del latibex. Sinapsis, v. 8, n. 2, 121-145. URL

Palomino Selem, C. (2010). El Mercado Integrado Latino Americano (MILA). Contexto, Hipótesis y Reflexiones. Pensamiento Crítico, v. 14, 119-135. https://doi.org/10.15381/pc.v14i0.9096

Peña Figueroa, I. (2019). Estudio de beneficios esperados en la integración de mercados accionarios emergentes. El caso de Latinoamerica. Revista cubana de ciencias económicas, v. 5, n. 2, 30 42. $\underline{U R L}$ 
Perafán Peña, H. F. (2018). Board gender diversity and earnings quality. Evidence from the Latin American integrated market (MILA). Contaduría Universidad de Antioquia, n. 72, 151-163. https://doi.org/10.17533/udea.rc.n72a08

Pérez Noreña, D., Giraldo Osorio, D. F., Gutiérrez Castañeda, B. E. (2020). Impacto del cambio de calificación de riesgo país en los precios de cotización de los activos de renta variable en el mercado integrado latinoamericano (MILA). Apuntes Contables, n. 26, 171-190. https://doi.org/10.18601/16577175.n26.10

Pérez Sosa, F. A., Gómez Hernández, D., Banda Ortiz, H. (2016). El mercado integrado latinoamericano (MILA): ¿̇oportunidad para los fondos de pensiones mexicanos? Denarius, $\mathrm{n}$. 30, 101-119. URL

Pineda Ospina, D. L. (2015). Análisis bibliométrico para la identificación de factores de innovación en la industria alimenticia. AD-minister, n. 27, 95-126. https://doi.org/10.17230/ad-minister.27.5

Ramírez, V. (2016). Efecto festivo y fin de semana en índices sectoriales del mercado chileno y peruano dentro del mercado integrado latinoamericano. Horizontes Empresariales, v. 15, n. 1, 61-74. URL

Reyes Zárate, F. J. (2016). Mercado Integrado Latinoamericano (MILA): un análisis de integración financiera y volatilidades. Estocástica: Finanzas y Riesgo, v. 6, n. 2, 187-218. URL

Rodríguez Aranda, I. (2014). Nuevas Configuraciones Económicas en el Asia-Pacífico y sus Consecuencias para América Latina: Desde el APEC a la Alianza del Pacífico. Dados - Revista de Ciências Sociais, v. 57, n. 2, 553-580. https://doi.org/10.1590/0011-5258201416

Rojas-Mora, J., Chamorro-Futinico, J. C. (2016). Dynamics and volatility at stock market indexes of Pacific Alliance countries. Panorama Económico, v. 24, n. 1, 71-84. URL

Romero-Álvarez, Y. P., Ramírez-Atehortúa, F. H., Guzmán-Aguilar, D. S. (2013). Mercado Integrado Latinoamericano (MILA): análisis de correlación y diversificación de los portafolios de acciones de los tres países miembros en el período 2007-2012. Cuadernos de Contabilidad, v. 14, n. 34, 53-74. URL

Ruiz Dávila, B. D., García Muñoz, G. (2020). Hipótesis de Mercados Eficientes y estrategias de inversión en el MILA: 2014-2019. Análisis Económico, v. 35, n. 90, 67-90. https://doi.org/10.24275/uam/azc/dcsh/ae/2020v35n90/Ruiz

Sancho, R. (1990). Indicadores bibliométricos utilizados en la evaluación de la ciencia y la tecnología: revisión bibliográfica. Revista Española de Documentacion Científica, v. 13, n. 3-4, 842-865. http://hdl.handle.net/10261/23694

Sandoval, E., Soto, M. (2016). Mercado Integrado Latinoamericano: Un Análisis De Cointegración. Revista Internacional Administración \& Finanzas, v. 9, n. 2, 1-17. URL 
Sandoval Álamos, E., Vásquez-Párraga, A. Z., Sabat Arriagada, R. (2015). Integración de los Mercados Accionarios de Chile, Colombia y Perú en el Mercado Integrado Latinoamericano (MILA). Innovar: Revista de ciencias administrativas y sociales, v. 25, n. 1Spe, 71-84. https://doi.org/10.15446/innovar.v25n1Spe.53195

Santillán-Salgado, R. J., Massa Roldán, R., Reyna Miranda, M. (2017). An Exploratory Study on Nonlinear Causality Among the MILA Markets. Emerging Markets Finance and Trade, v. 53, n. 10, 2303-2317. https://doi.org/10.1080/1540496X.2017.1308861

Sosa, M., Ortiz, E., Cabello, A. (2018). Dynamic Linkages between Stock Market and Exchange Rate in mila Countries: A Markov Regime Switching Approach (2003-2016). Análisis económico, v. 33, n. 83, 57-74. https://doi.org/10.24275/uam/azc/dcsh/ae/2018v33n83/sosa

Suárez-Rico, Y. M., García-Benau, M. A., Gómez-Villegas, M. (2019). CSR communication through Facebook in the Latin American integrated market (MILA): Disclosure, interactivity and legitimacy. Meditari Accountancy Research, v. 27, n. 5, 741-760.

https://doi.org/10.1108/MEDAR-03-2018-0313

Torres-Cano, S. M., Correa-Mejía, D. A. (2021). Impacto del gobierno corporativo en el valor de las empresas latinoamericanas: evidencia desde el MILA. Suma de Negocios, v. 12, n. 26, 73-82. http://doi.org/10.14349/sumneg/2021.V12.N26.A8

Uribe Gil, J. M., Mosquera López, S. (2014). Efectos del MILA en la eficiencia de portafolio de los mercados de acciones colombiano, peruano y chileno. Cuadernos de Administración, v. 30, n. 52, 75-83. $\underline{U R L}$

Vargas Pulido, W., Bayardo Martínez, J. B. (2013). El MILA. Mercado de integración entre Chile, Perú y Colombia. Revista de Relaciones Internacionales, Estrategia y Seguridad, v. 8, n. 1, 113-133. https://doi.org/10.18359/ries.67

Venable, G., Khan, N., Taylor, D., Thompson, T., Michael, L., Klimo Jr., P. (2014). A Correlation Between National Institutes of Health Funding and Bibliometrics in Neurosurgery. World Neurosurgery, v. 81, n. 3-4, 468-472. http://doi.org/10.1016/j.wneu.2013.11.013

Yepes-Ríos, B., González-Tapia, K., González-Pérez, M. A. (2015). The integration of stock exchanges: The case of the Latin American Integrated Market (MILA) and its impact on ownership and internationalization status in Colombian brokerage firms. Journal of Economics, Finance and Administrative Science, v. 20, n. 39, 84-93. http://doi.org/10.1016/j.jefas.2015.08.001

Zamarra Londoño, J. E., Gutiérrez Castañeda, B. E., Pérez Noreña, D. (2021). Análisis de la información financiera en torno al informe del auditor de las empresas del MILA. Suma de Negocios, v. 12, n. 26, 64-72. http://doi.org/10.14349/sumneg/2021.V12.N26.A7

Zamarra-Londoño, J., Pérez-Noreña, D., Pareja-Taborda, A. (2020). El informe de auditoría en los países del MILA. Desarrollo Gerencial, v. 12, n. 1, 1-20.

http://doi.org/10.17081/dege.12.1.3783 